\title{
A multiwavelength study of star formation in the very young open cluster NGC 6530*
}

\author{
M.E. van den Ancker ${ }^{1}$, P.S. Thé ${ }^{1}$, A. Feinstein ${ }^{2,3,4}$, R.A. Vázquez ${ }^{2,3}$, D. de Winter ${ }^{1,5}$, and M.R. Pérez ${ }^{6}$ \\ 1 Astronomical Institute "Anton Pannekoek", University of Amsterdam, Kruislaan 403, NL-1098 SJ Amsterdam, \\ The Netherlands \\ 2 Observatorio Astronómico de La Plata, Paseo del Bosque, 1900 La Plata, Argentina \\ 3 Member of Carrera del Investigador Cientifico, CONICET, Buenos Aires, Argentina \\ 4 Visiting astronomer, Cerro Tololo Inter-American observatory, operated under contract No. AST 78-27879 \\ 5 Dpto. Física Téorica C-XI, Facultad de Ciencias, Universidad Autónoma de Madrid, Cantoblanco, E-28049 Madrid, Spain \\ 6 Applied Research Corporation, Suite 1120, 8201 Corporate Dr., Landover MD 20785, U.S.A.
}

Received April 18; accepted September 11, 1996

\begin{abstract}
The distance and formation history of the very young open cluster NGC 6530 were investigated by studying a total of 132 probable member stars of this open cluster. It was concluded that the distance to NGC 6530 is $1.8 \pm 0.2 \mathrm{kpc}$, and its interstellar reddening $E(B-V)$ is 0.30 . Furthermore, we conclude that the extinction law of the intracluster material is normal (i.e. $R_{V}=3.1$ ), but anomalous extinction laws were found for several more embedded stars in the cluster. Of the 132 stars included in this study, 11 are suspected to be variable, five show $\mathrm{H} \alpha$ in emission and nine show an infrared excess. Among the member stars of NGC 6530 , three were found to be part of the Herbig Ae/Be stellar class, whereas two others are possible members of this stellar group as well. Also, one cluster member is probably a new massive post-AGB star, whereas the same could possibly apply to another member star. Finally, from the distribution of post- and pre-main sequence stars in the cluster's HR-diagram, it was concluded that the process of star formation in NGC 6530 must have started a few times $10^{7}$ years ago and, for the less massive stars, is probably still going on today.
\end{abstract}

Key words: circumstellar matter - stars: formation stars: pre-main sequence - dust, extinction - H II regions - open clusters and associations: NGC 6530

Send offprint requests to: M.E. van den Ancker (mario@astro.uva.nl)

* Based on observations collected at the European Southern Observatory, La Silla, Chile, on observations collected at Cerro Tololo Inter-American Observatory, and on spectral data obtained with the International Ultraviolet Explorer. Tables 1, 2 and 5 are available in electronic form at the CDS via anonymous ftp to cdsarc.u-strasbg.fr (130.79.128.5) or via http://cdsweb.u-strasbg.fr/Abstract.html

\section{Introduction}

NGC 6530 (C1801-243, in the central part of the H II region $\mathrm{M} 8$, the Lagoon nebula) is one of the most studied open clusters in our galaxy. Together with many other clusters and nebulae like NGC 6531, 6611, 6613, M 17 and M 20 it forms the star forming region SFR Sgr I (Stal'bovskii \& Shevchenko 1981). Walker (1957) concluded, from $U B V$ photoelectric observations of 118 stars in NGC 6530 , that the colour-magnitude diagram of this cluster consists of a normal main sequence extending from O5 to about A0, with stars of later spectral type lying above the main sequence. From this he concluded that the cluster age must be about $310^{6}$ years. Walker also suggested that the reddening in NGC 6530 might be anomalous, which was confirmed for a small number of stars by the results of Johnson (1967).

Thé (1960) argued, on the basis of star counts, that a significant fraction of Walker's pre-main sequence stars must be foreground or background stars. This was later confirmed by a proper motion study of 363 stars (complete up to $V \approx 13 .{ }^{\mathrm{m}} 6$ ) in the vicinity of NGC 6530 by van Altena \& Jones (1972). Later Chini \& Neckel (1981) demonstrated that the bulk of the probable members from these proper motion studies are also located on the main sequence.

A model for the structure of the $\mathrm{M} 8$ region was given by Lada et al. (1976), who proposed that NGC 6530 was formed from a pre-existent massive molecular cloud, in which it produced a "blister" due to the ionizing radiation of the newly formed hot stars. Because of this cavity we can look further into the molecular cloud and we can also see the more recently formed embedded stars. Lightfoot et al. (1984) used this model to explain the star formation in $\mathrm{M} 8$ as occurring in three generations: first to form were the hot stars in NGC 6530 . These triggered 
Table 1. Properties of programme stars in NGC 6530 from literature

\begin{tabular}{|c|c|c|c|c|c|c|c|c|c|c|c|c|}
\hline \multicolumn{3}{|c|}{ Star number } & \multirow{2}{*}{$\begin{array}{l}\text { Other } \\
\text { designation }\end{array}$} & \multirow[t]{2}{*}{$\alpha(1950)$} & \multirow[t]{2}{*}{$\delta(1950)$} & \multirow{2}{*}{$\begin{array}{c}P \\
{[\%]}\end{array}$} & \multirow[t]{2}{*}{$V$} & \multirow[t]{2}{*}{$B-V$} & \multirow[t]{2}{*}{$U-B$} & \multirow{2}{*}{$\begin{array}{c}v_{\mathrm{r}} \\
{\left[\mathrm{km} \mathrm{s}^{-1}\right]}\end{array}$} & \multirow{2}{*}{$\begin{array}{l}\text { Spectral } \\
\text { type }\end{array}$} & \multirow[t]{2}{*}{ Ref } \\
\hline$\overline{\text { VAJ }}$ & Walker & Sagar & & & & & & & & & & \\
\hline 1 & & & & 175917.6 & -241504 & 12 & 13.07 & & & & & \\
\hline 2 & & & & 175918.7 & -242543 & 46 & 13.15 & & & & & \\
\hline 28 & 3 & 1 & $\mathrm{CD}-24^{\circ} 13785$ & 175935.8 & -241452 & 72 & 8.66 & 0.00 & -0.75 & & & \\
\hline 29 & 212 & & & 175938.7 & -241728 & 32 & 12.70 & 0.66 & 0.50 & & & \\
\hline 30 & 211 & 2 & & 175939.0 & -241816 & 64 & 12.40 & 0.14 & -0.13 & & & \\
\hline 35 & 210 & 3 & & 175942.9 & -242036 & 76 & 12.26 & 0.22 & 0.07 & & & \\
\hline 38 & 300 & & & 175944.3 & -241446 & 13 & 13.52 & 1.00 & 0.04 & & & \\
\hline 45 & 303 & 4 & & 175946.9 & $-24 \quad 1623$ & 85 & 12.04 & 0.62 & 0.26 & & & \\
\hline 47 & & 5 & & 175947.4 & -243737 & 65 & 12.65 & 0.39 & 0.26 & & & \\
\hline 53 & & 6 & & 175949.9 & -240952 & 78 & 11.43 & 1.05 & 0.04 & & & \\
\hline 55 & & & & 175950.2 & -243747 & 10 & 13.28 & & & & & \\
\hline 72 & & 7 & & 180001.8 & -240746 & 50 & 13.49 & 0.44 & 0.15 & & & \\
\hline 73 & 219 & 8 & & 180002.4 & -241549 & 72 & 12.39 & 0.50 & 0.22 & & & \\
\hline 74 & 200 & 9 & & 180002.8 & -242446 & 70 & 12.77 & 0.32 & 0.21 & & & \\
\hline 79 & & 10 & & 180010.2 & -240735 & 69 & 12.66 & 1.32 & 1.06 & & & \\
\hline 89 & 282 & & & 180021.5 & -240946 & 15 & 13.49 & 0.94 & -0.17 & & & \\
\hline 91 & 181 & 11 & & 180021.9 & -242243 & 78 & 12.90 & 0.56 & 0.09 & & & \\
\hline 92 & 244 & 12 & & 180022.3 & -241340 & 85 & 12.03 & 1.20 & 1.08 & & & \\
\hline 93 & & 13 & & 180022.6 & -240634 & 83 & 11.70 & 0.32 & -0.25 & & & \\
\hline 94 & 241 & 14 & & 180022.8 & -241254 & 80 & 11.91 & 0.20 & -0.27 & & & \\
\hline 97 & 178 & 15 & & 180024.4 & -242311 & 45 & 10.86 & 1.20 & 1.32 & & K4 III & 1 \\
\hline 98 & 239 & & & 180025.6 & -241329 & 10 & 13.72 & 0.72 & -0.01 & & & \\
\hline 100 & & 16 & & 180027.2 & -243644 & 59 & 11.89 & 1.25 & 1.19 & & & \\
\hline 107 & 170 & 17 & & 180041.0 & -242215 & 86 & 10.05 & 0.13 & -0.51 & & $\mathrm{~B} 2.5 \mathrm{~V}$ & 1 \\
\hline 113 & 172 & 18 & & 180046.5 & -242110 & 78 & 12.15 & 0.29 & -0.46 & & & \\
\hline 114 & 173 & 19 & $\mathrm{LkH} \alpha 108$ & 180046.7 & -242121 & 77 & 12.78 & 0.37 & -0.17 & & & \\
\hline 118 & 123 & & & 180049.2 & -243151 & 47 & 13.58 & 0.88 & -0.32 & & & \\
\hline \multirow[t]{3}{*}{120} & 168 & 20 & & 180050.2 & -242315 & 62 & 11.84 & 0.46 & -0.23 & & & \\
\hline & 9 & & HD 164816 & 180052.9 & -241856 & & 7.11 & 0.01 & -0.84 & & B0 V & 2 \\
\hline & 13 & & & 180055 & -241517 & & 13.34 & 0.46 & 0.15 & & & \\
\hline 132 & 14 & 22 & & 180057.1 & -242640 & 41 & 11.49 & 0.13 & -0.32 & & B5.5 V & 1 \\
\hline 133 & 15 & 23 & & 180057.1 & -242358 & 57 & 11.67 & 0.10 & -0.29 & & B7 & 3 \\
\hline 135 & 16 & 24 & & 180058.9 & -241106 & 50 & 12.02 & 0.31 & 0.13 & & & \\
\hline 137 & 121 & 25 & & 180059.9 & -242842 & 79 & 13.87 & 0.59 & -0.33 & & & \\
\hline 138 & 17 & 26 & & 180100.2 & -242141 & 77 & 12.04 & 0.08 & -0.27 & & B7 V & 1 \\
\hline 141 & 260 & 28 & & 180103.4 & -242239 & 34 & 13.26 & 1.14 & 0.64 & & & \\
\hline 142 & 163 & 29 & & 180104.0 & -241255 & 69 & 12.72 & 0.22 & 0.11 & & & \\
\hline 146 & 25 & 30 & & 180105.3 & -241554 & 86 & 11.49 & 0.09 & -0.29 & & & \\
\hline 147 & 26 & 31 & & 180105.8 & -242020 & 62 & 11.64 & 0.20 & -0.26 & & A0 & 3 \\
\hline 149 & 24 & 32 & & 180105.9 & -242422 & 64 & 11.96 & 0.11 & -0.21 & & B9: & 3 \\
\hline 150 & 27 & 33 & & 180106.2 & -242103 & 79 & 12.96 & 0.63 & 0.38 & & F5-G0 & 4 \\
\hline 151 & 29 & & & 180107.1 & -242459 & 20 & 13.15 & 0.80 & -0.19 & & F0-G5 & 4 \\
\hline 152 & 32 & 34 & & 180107.1 & -242156 & 84 & 10.43 & 0.10 & -0.54 & & $\mathrm{~B} 3 \mathrm{Ve}$ & 5 \\
\hline 154 & 33 & 35 & & 180107.3 & -241635 & 52 & 13.30 & 0.79 & 0.45 & & & \\
\hline 155 & 31 & 36 & & 180107.4 & -242541 & 52 & 11.69 & 0.23 & -0.25 & & A0 & 3 \\
\hline 157 & 38 & 37 & & 180110.0 & -241340 & 68 & 12.16 & 0.49 & 0.35 & & & \\
\hline \multirow[t]{2}{*}{161} & 42 & 38 & HD 315032 & 180110.9 & -242339 & 84 & 9.19 & 0.02 & -0.73 & -19.0 & $\mathrm{~B} 1 \mathrm{~V}$ & 2 \\
\hline & 43 & & HD 315026 & 180111.0 & -241443 & & 9.01 & 0.08 & -0.61 & & $\mathrm{~B} 1.5 \mathrm{~V}$ & 2 \\
\hline 163 & 47 & 39 & ADS $11015 \mathrm{D}$ & 180111.8 & -241507 & 76 & 12.24 & 0.21 & 0.00 & & & \\
\hline 165 & 46 & 40 & & 180112.5 & -242849 & 60 & 11.62 & 0.18 & -0.17 & & A0 & 3 \\
\hline 166 & 49 & & & 180112.6 & -241728 & 15 & 11.10 & 0.16 & -0.44 & & F5: & 3 \\
\hline 170 & & & & 180115.1 & -243521 & 18 & 13.02 & 0.56 & 0.42 & & & \\
\hline 171 & 52 & 41 & & 180115.6 & -241447 & 85 & 12.12 & 0.30 & 0.20 & & & \\
\hline 172 & 126 & & & $1801 \quad 15.7$ & -243013 & 19 & 11.70 & 1.35 & 1.14 & & & \\
\hline 173 & 53 & 42 & & 180116.4 & -242507 & 78 & 13.11 & 0.57 & 0.47 & & G0: & 4 \\
\hline
\end{tabular}

the formation of the O4f star $9 \mathrm{Sgr}$, located deeper into the molecular cloud, which in turn has caused the formation of the even younger protostar Herschel 36, located the furthest away from the sun.

There seems to be consensus between the different authors on the distance and interstellar reddening towards NGC 6530. Walker (1957) already obtained a distance be- tween 1.4 and $2.0 \mathrm{kpc}$ and an $E(B-V)$ between 0.33 and $0{ }^{\mathrm{m}} 37$ for NGC 6530 . These results were confirmed by most subsequent authors, although Sagar \& Joshi (1978) found $E(B-V)$ to vary from 0.25 to 0.48 across the cluster. The most recent values for $E(B-V)$ and the distance are 0. $36 \pm 0$ m 09 by Chini \& Neckel (1981) and $1.86 \pm 0.07 \mathrm{kpc}$ by McCall et al. (1990). 
Table 1. continued

\begin{tabular}{|c|c|c|c|c|c|c|c|c|c|c|c|c|}
\hline \multicolumn{3}{|c|}{ Star number } & \multirow{2}{*}{$\begin{array}{l}\text { Other } \\
\text { designation }\end{array}$} & \multirow[t]{2}{*}{$\alpha(1950)$} & \multirow[t]{2}{*}{$\delta(1950)$} & \multirow{2}{*}{$\begin{array}{c}P \\
{[\%]}\end{array}$} & \multirow[t]{2}{*}{$V$} & \multirow[t]{2}{*}{$B-V$} & \multirow[t]{2}{*}{$U-B$} & \multirow{2}{*}{$\begin{array}{c}v_{\mathrm{r}} \\
{\left[\mathrm{km} \mathrm{s}^{-1}\right]}\end{array}$} & \multirow{2}{*}{$\begin{array}{l}\text { Spectral } \\
\text { type }\end{array}$} & \multirow[t]{2}{*}{ Ref } \\
\hline$\overline{\text { VAJ }}$ & Walker & Sagar & & & & & & & & & & \\
\hline 176 & 56 & 43 & $\mathrm{CD}-24^{\circ} 13829$ & 180117.2 & -242130 & 86 & 9.08 & 0.09 & -0.70 & -8.4 & B0.5 V & 2 \\
\hline 177 & 54 & 44 & & 180117.2 & -242347 & 62 & 11.56 & 0.25 & 0.08 & & & \\
\hline 180 & 58 & 45 & LkH $\alpha 112$ & 180118.6 & -242222 & 81 & 9.78 & 0.26 & -0.63 & & $\mathrm{~B} 2 \mathrm{Ve}$ & 5 \\
\hline 181 & & 46 & & 180118.7 & -243630 & 61 & 12.51 & 0.42 & 0.40 & & & \\
\hline 182 & 59 & 47 & HD 315033 & 180119.0 & -242629 & 75 & 8.94 & 0.10 & -0.62 & -22 & B1 V & 6 \\
\hline 183 & 316 & & & 180119.5 & -242129 & 45 & 12.56 & 0.98 & 0.36 & & & \\
\hline 184 & 60 & 48 & & 180119.9 & -242139 & 81 & 9.64 & 0.11 & -0.62 & -4.3 & $\mathrm{~B} 1 \mathrm{Ve}$ & 5 \\
\hline 185 & 61 & & & 180120.2 & -242112 & 25 & 10.29 & 0.12 & -0.61 & & $\mathrm{~B} 2 \mathrm{Ve}$ & 5 \\
\hline 187 & 263 & 49 & & 180120.8 & -242757 & 70 & 11.40 & 0.20 & -0.32 & & & \\
\hline 189 & 291 & 50 & & 180121.0 & -240930 & 61 & 13.18 & 1.31 & 0.72 & & & \\
\hline 190 & 64 & 51 & & 180121.2 & -242254 & 48 & 11.65 & 0.17 & -0.31 & & B5 V & 1 \\
\hline 192 & 66 & 52 & $\mathrm{CD}-24^{\circ} 13831$ & 180121.5 & -242057 & 79 & 10.16 & 0.11 & -0.61 & -12.0 & B2 Vpe & 5 \\
\hline 194 & 67 & 54 & & 180121.7 & -242002 & 86 & 11.87 & 0.45 & 0.03 & & & \\
\hline 195 & 160 & 55 & & 180122.6 & -242404 & 74 & 12.33 & 0.48 & 0.12 & & & \\
\hline 197 & 70 & 56 & & 180123.1 & -242302 & 83 & 10.52 & 0.11 & -0.54 & & $\mathrm{~B} 2 \mathrm{Ve}$ & 5 \\
\hline 199 & 71 & & & 180123.2 & -241958 & 22 & 13.27 & 1.07 & 0.27 & & & \\
\hline 201 & 73 & 57 & HD 315031 & 180123.9 & -242155 & 48 & 8.30 & 0.08 & -0.70 & -13 & B1 V & 2 \\
\hline 202 & 74 & 58 & & 180124.8 & -242539 & 86 & 10.67 & 0.11 & -0.52 & & $\mathrm{~B} 2.5 \mathrm{Ve}$ & 2 \\
\hline 203 & 77 & 59 & & 180125.0 & -241415 & 83 & 11.99 & 0.45 & 0.52 & & & \\
\hline 204 & 76 & 60 & HD 315024 & 180125.2 & -241937 & 85 & 9.53 & 0.05 & -0.68 & -18.8 & $\mathrm{~B} 2.5 \mathrm{Ve}$ & 5 \\
\hline 205 & & & & 180125.5 & -240626 & 49 & 13.13 & 0.65 & 0.07 & & & \\
\hline 206 & 151 & & & 180125.6 & -240948 & 49 & 12.19 & 0.72 & 0.49 & & & \\
\hline \multirow[t]{2}{*}{207} & 131 & 61 & & 180127.0 & -243233 & 66 & 13.12 & 0.33 & 0.13 & & & \\
\hline & 80 & & $\mathrm{CD}-24^{\circ} 13837$ & 180128.1 & -242319 & & 9.39 & 0.07 & -0.72 & -10.9 & B1 V & 2 \\
\hline 210 & 83 & 62 & LSS 4621 & 180128.9 & -241857 & 75 & 10.44 & 0.11 & -0.55 & & B2.5 Vne & 5 \\
\hline 211 & 81 & 63 & & 180128.9 & -242306 & 59 & 11.72 & 0.17 & -0.47 & & & \\
\hline 212 & 82 & 64 & & 180129.0 & -242128 & 70 & 12.44 & 0.29 & -0.22 & & & \\
\hline 215 & 86 & 65 & $\mathrm{CD}-24^{\circ} 13840$ & 180130.1 & -242213 & 77 & 9.88 & 0.13 & -0.46 & & $\mathrm{~B} 2.5 \mathrm{~V}$ & 2 \\
\hline 216 & 84 & 66 & & 180130.2 & -242818 & 79 & 11.89 & 0.20 & -0.26 & & A0 & 3 \\
\hline 223 & 93 & 67 & HD 315021 & 180131.9 & -242005 & 84 & 8.57 & 0.05 & -0.73 & -14.6 & B1 V & 2 \\
\hline 226 & 94 & & & 180133.6 & -242125 & 25 & 12.65 & 0.26 & -0.02 & & A: & 3 \\
\hline 228 & 96 & 68 & & 180134.9 & -242311 & 55 & 12.18 & 0.23 & -0.14 & & & \\
\hline 229 & 97 & 69 & & 180134.9 & -241939 & 77 & 11.39 & 0.12 & -0.46 & & B9 & 3 \\
\hline \multirow[t]{2}{*}{230} & 99 & 70 & $\mathrm{CD}-24^{\circ} 13844$ & 180135.8 & -242258 & 50 & 10.81 & 0.09 & -0.52 & & B2.5 Vne & 5 \\
\hline & 100 & & HD 164947 & 180137.7 & -242108 & & 8.88 & 0.06 & -0.56 & +6.1 & $\mathrm{~B} 2.5 \mathrm{~V}$ & 2 \\
\hline 235 & 105 & 71 & HD 315022 & 180138.8 & -241847 & 56 & 10.53 & 0.12 & -0.29 & & B6 V & 1 \\
\hline 238 & 134 & 72 & & 180141.7 & -242733 & 37 & 12.67 & 0.80 & 0.20 & & & \\
\hline 239 & 111 & 73 & & 180143.4 & -241815 & 58 & 12.60 & 0.23 & -0.03 & & & \\
\hline 240 & 110 & 74 & & 180143.5 & -242838 & 70 & 11.23 & 0.15 & -0.45 & & B3 V & 1 \\
\hline 242 & 314 & 75 & & 180144.4 & -242649 & 85 & 13.24 & 1.06 & 0.25 & & & \\
\hline 244 & 145 & & & 180145.3 & -241322 & 12 & 13.42 & 0.81 & 0.33 & & & \\
\hline 245 & 114 & 76 & $\mathrm{LkH} \alpha 115$ & 180146.4 & -242556 & 79 & 11.98 & 0.32 & -0.12 & & $\mathrm{~B} 4 \mathrm{Ve}$ & 7 \\
\hline 246 & 116 & 77 & HD 315020 & 180148.7 & -241807 & 52 & 11.28 & 0.26 & 0.22 & & B8 & 3 \\
\hline 247 & 158 & 78 & & 180149.0 & -241942 & 81 & 11.51 & 0.28 & -0.40 & & & \\
\hline 250 & 137 & 79 & HD 315034 & 180152.1 & -242418 & 86 & 11.59 & 0.26 & -0.36 & & B3 V & 1 \\
\hline 256 & 117 & 80 & HD 315035 & 180154.8 & -242738 & 71 & 10.80 & 0.22 & -0.36 & & B3 V & 2 \\
\hline 258 & 140 & & & 180157.7 & -241853 & 46 & 13.05 & 0.36 & 0.44 & & & \\
\hline 259 & 141 & 81 & $\mathrm{CD}-24^{\circ} 13858$ & 180158.3 & $-24 \quad 17 \quad 17$ & 84 & 11.55 & 0.35 & -0.03 & & & \\
\hline 261 & 139 & 82 & & 180200.0 & -242524 & 74 & 12.24 & 1.34 & 1.50 & & & \\
\hline 266 & 142 & 83 & $\mathrm{CD}-24^{\circ} 13862$ & 180204.3 & -241548 & 80 & 11.71 & 0.20 & -0.34 & & & \\
\hline 269 & & 84 & & 180206.0 & -243224 & 82 & 12.50 & 0.71 & 0.23 & & & \\
\hline 270 & 118 & & HD 165052 & 180206.5 & -242411 & 39 & 6.88 & 0.10 & -0.81 & +3.0 & $\mathrm{O} 6.5+\mathrm{O} 6.5 \mathrm{~V}$ & 2 \\
\hline 272 & & & & 180207.2 & -242453 & 44 & 13.10 & & & & & \\
\hline 275 & & 85 & & 180210.1 & -240617 & 70 & 13.02 & 0.39 & 0.30 & & & \\
\hline 284 & & 86 & & 180219.2 & -242021 & 52 & 13.10 & 0.67 & 0.26 & & & \\
\hline
\end{tabular}

Because Chini \& Neckel (1981) neglected the effects of anomalous extinction and used only very rough spectral classifications from $U B V$ photometry we concluded that a new study of this cluster, taking into account these effects, might yield different results. Therefore, we selected all stars from the proper motion study by van Altena $\&$ Jones (1972) with membership probabilities $\geq 10 \%$, together with five additional stars from the original paper by Walker (1957), which were not included in their study, to investigate this interesting cluster further using new photometric and spectroscopic data. Properties of all programme stars, obtained from literature, are listed in Table 1. In the remainder of this paper these stars will be indicated by their number in the paper by van Altena 
Table 1. continued

\begin{tabular}{|c|c|c|c|c|c|c|c|c|c|c|c|c|}
\hline \multicolumn{3}{|c|}{ Star number } & \multirow{2}{*}{$\begin{array}{l}\text { Other } \\
\text { designation }\end{array}$} & \multirow[t]{2}{*}{$\alpha(1950)$} & \multirow[t]{2}{*}{$\delta(1950)$} & \multirow{2}{*}{$\begin{array}{c}P \\
{[\%]}\end{array}$} & \multirow[t]{2}{*}{$V$} & \multirow[t]{2}{*}{$B-V$} & \multirow[t]{2}{*}{$U-B$} & \multirow{2}{*}{$\begin{array}{c}v_{\mathrm{r}} \\
{\left[\mathrm{km} \mathrm{s}^{-1}\right]}\end{array}$} & \multirow{2}{*}{$\begin{array}{l}\text { Spectral } \\
\text { type }\end{array}$} & \multirow[t]{2}{*}{ Ref } \\
\hline$\overline{\text { VAJ }}$ & Walker & Sagar & & & & & & & & & & \\
\hline 286 & & & & 180226.1 & -241113 & 18 & 13.39 & 0.77 & -0.03 & & & \\
\hline 296 & & 87 & & 180235.5 & -240810 & 83 & 13.31 & 0.65 & 0.11 & & & \\
\hline 298 & & 88 & & 180239.1 & -242928 & 63 & 12.58 & 0.43 & 0.20 & & & \\
\hline 301 & & 89 & & 180248.2 & -243403 & 37 & 11.91 & 1.33 & 1.37 & & & \\
\hline 304 & & 90 & & 180250.5 & -241517 & 73 & 9.92 & 2.29 & 3.14 & & & \\
\hline 306 & & 91 & & 180251.9 & -241411 & 81 & 13.33 & 1.40 & 0.82 & & & \\
\hline 309 & & 92 & & 180254.1 & -242600 & 33 & 11.70 & 0.76 & 0.49 & & & \\
\hline 315 & & 93 & & 180257.5 & -241458 & 35 & 13.10 & 0.72 & 0.14 & & & \\
\hline 316 & & & & 180257.6 & -241807 & 13 & 13.55 & & & & & \\
\hline 317 & & 94 & & 180257.7 & -241356 & 85 & 11.94 & 0.78 & 0.18 & & & \\
\hline 329 & & & & 180307.3 & -242412 & 13 & 12.18 & 0.53 & -0.02 & & & \\
\hline 330 & & 95 & & $1803 \quad 07.5$ & -243122 & 45 & 10.75 & 0.79 & 0.19 & & & \\
\hline 331 & & & & 180307.6 & -241941 & 10 & 12.77 & 0.65 & 0.29 & & & \\
\hline 332 & & 96 & & 180307.8 & -242040 & 80 & 13.47 & 0.63 & 0.39 & & & \\
\hline 336 & & 97 & & 180313.6 & -242258 & 78 & 13.13 & 0.56 & 0.31 & & & \\
\hline 337 & & & & 180314.1 & -241618 & 19 & 13.26 & 0.81 & 0.22 & & & \\
\hline 338 & & 98 & & 180315.8 & -241040 & 69 & 10.26 & 2.32 & 2.41 & & & \\
\hline 342 & & & & 180318.5 & -242831 & 12 & 11.84 & 1.12 & 0.93 & & & \\
\hline 347 & & & & 180322.0 & -242103 & 10 & 12.77 & 0.58 & 0.13 & & & \\
\hline 353 & & 99 & & 180327.0 & -243412 & 50 & 12.02 & 0.46 & 0.29 & & & \\
\hline 360 & & 100 & & 180335.2 & -243819 & 74 & 12.10 & 0.74 & 0.34 & & & \\
\hline 361 & & 101 & & 180336.1 & -243446 & 58 & 12.84 & 0.44 & 0.28 & & & \\
\hline
\end{tabular}

References: (1) Buscombe (1984); (2) Boggs \& Böhm-Vitense (1989); (3) Walker (1957); (4) Johnson \& Borgman (1963); (5) Hiltner et al. (1965); (6) Torres (1987); (7) Boesono et al. (1987); (8) Morrison \& Conti 1978.

W 43. Triple system (Torres 1987).

171. Variable star (Sagar \& Joshi 1978).

176. Binary (Boggs \& Böhm-Vitense 1989).

180. Herbig Be star (Boesono et al. 1987).

184. Radial velocity probably variable. Perhaps double lines in the spectrum (Walker 1957).

192. He I star (Hiltner et al. 1965).

201. Binary system, with a secondary of the same spectral type as the primary (Boggs \& Böhm-Vitense 1989).

W 80. Radial velocity probably variable. Perhaps double lines in the spectrum (Walker 1957).

215. Double-lined spectroscopic binary (Hiltner et al. 1965).

W 100. Binary system, with a secondary of the same spectral type as the primary (Boggs \& Böhm-Vitense 1989).

242. Walker's variable star No. 64.

245. Herbig Be star (Boesono et al. 1987). Walker's variable star No. 68.

270. Double-lined spectroscopic binary, consisting of two O6.5 V stars with a period of 6. 140 (Morrison \& Conti 1978).

284. Variable star (Sagar \& Joshi 1978).

338. Variable star (Sagar \& Joshi 1978).

353. Photometric variable (van Altena \& Jones 1972).

\& Jones (1972), also listed in the first column of Table 1. The five programme stars which were not included in this paper will be indicated by a $\mathrm{W}$ followed by their number in the paper by Walker (1957) which is listed in the second column of Table 1. The star numbers higher than 118 in this column are from the extension of this numbering system by Kilambi (1977). The third column in Table 1 lists the corresponding stellar numbers from the paper by Sagar \& Joshi (1978).

\section{The observations}

\subsection{Photometry}

New photometric data in the Walraven $W U L B V$ system of our programme stars were obtained during three observing runs, in May 1982, June 1983, and in August 1985 us- ing the $90 \mathrm{~cm}$ Dutch Light Collector at La Silla. Measuring and reduction procedures were the same as described in Lub \& Pel (1977), except that a 16.5 diaphragm was used. In order to compare these data with the photometric data from literature in the Johnson $U B V$ system we transformed $V$ intensities in the Walraven $W U L B V$ system to Johnson $V$ magnitudes using the formula quoted in Brand \& Wouterloot (1988):

$$
V_{\mathrm{J}}=6.884-2.5\left\{V_{\mathrm{W}}+0.039 \times(V-B)_{\mathrm{W}}\right\} .
$$

These transformed $V_{\mathrm{J}}$ magnitudes are listed in the third column of Table 2.

Photometric data in the Johnson/Cousins $U B V(R I)_{\mathrm{C}}$ system of our programme stars in NGC 6530 were obtained during four observing runs, in May 1984 at the Cerro Tololo Inter-American Observatory (CTIO), and in June 1983, July 1985 and March 1991 at the European 
Southern Observatory (ESO), La Silla, Chile. At CTIO the observations were carried out using a $16^{\prime \prime}$ diaphragm at the $60 \mathrm{~cm}$ Lowell telescope, equipped with an RCA 31034A (Quantacon) photomultiplier. At La Silla the observations were made with the ESO $50 \mathrm{~cm}$ telescope equipped with an identical photomultiplier and a $15^{\prime \prime}$ diaphragm. Sky subtraction was achieved by subtracting sky measurements $30^{\prime \prime}$ east from the star, unless that position showed a significantly higher than background flux, in which case a sky measurement $30^{\prime \prime}$ west of the star was obtained and used. About 35 E-region standard stars from the list of Graham (1982) were observed each night and used for the determination of the extinction and colour transformation parameters. In this way the obtained typical errors for stars brighter than 12.5 are 0 . 01 for $V, B-V, V-R$ and $V-I$, and 0.02 for $U-B$. For fainter stars these results are about 0.01 less certain. However, since the observed set of E-region standards for the observing runs at ESO in 1983 and 1985 did not include stars later than F6, transformation errors for stars with very red colours can be much larger in these data. In order to compare our new $U B V$ photometric data with those measured by previous authors we computed the average difference between our measurements and those by Chini \& Neckel (1981), with $\Delta V=\overline{V_{\mathrm{C} \& \mathrm{~N}}-V}$, together with the same difference between their data set and those by other authors. Omitted from this systematic difference calculation were 11 stars with differences larger than 0.30 in $V$ between the various data sets. For stars which were only measured 2 or 3 times this difference might be due to stellar misidentifications, whereas for stars which were measured more often these stars are probably real variables. These 11 suspected variable stars are listed in Table 3 . The systematic differences between our data set as well as several data sets from literature with the one by Chini \& Neckel (1981) are listed in Table 4. In cases where this systematic difference is significant (i.e. the difference is larger than its standard deviation listed in Table 4), the data have been corrected for this in the remainder of this paper.

Near-IR $J H K$ photometric data of stars in NGC 6530 were obtained in July 1986 with the ESO $1 \mathrm{~m}$ telescope at La Silla, equipped with an InSb detector. These observations were made through a $15^{\prime \prime}$ diaphragm. Sky subtraction was achieved by chopping, with a frequency of $8 \mathrm{~Hz}$, in the east-west direction with a throw of $30^{\prime \prime}$ amplitude. About 35 standard stars from a preliminary version of the list later published by Bouchet et al. (1989) were observed each night and used for the determination of the extinction parameters. Typical errors in the thus data are about 0.05 for the $J, H$ and $K$ magnitudes. All new photometric measurements are listed in Table 2.
Table 3. Suspected variable stars in NGC $6530\left(\Delta V>0 .{ }^{\mathrm{m}} 30\right)$

\begin{tabular}{|c|c|c|c|c|c|c|c|}
\hline \multirow[t]{2}{*}{ No. } & \multicolumn{2}{|c|}{$V$} & \multicolumn{2}{|c|}{$B-V$} & \multicolumn{2}{|c|}{$U-B$} & \multirow[t]{2}{*}{$n$} \\
\hline & min. & $\max$ & min. & $\max$ & min. & $\max$ & \\
\hline 45 & 12.04 & 13.71 & 0.62 & 0.74 & +0.20 & +0.26 & $\sigma$ \\
\hline 91 & 12.66 & 13.02 & 0.54 & 0.56 & -0.11 & +0.09 & 3 \\
\hline 114 & 11.69 & 12.78 & 0.21 & 0.41 & -0.27 & -0.06 & 3 \\
\hline 120 & 11.84 & 12.16 & 0.25 & 0.46 & -0.23 & -0.10 & 3 \\
\hline 151 & 13.14 & 13.68 & 0.80 & 0.83 & -0.19 & +0.21 & 7 \\
\hline 171 & 11.89 & 12.27 & 0.26 & 0.31 & -0.05 & +0.23 & 6 \\
\hline 180 & 9.54 & 10.19 & 0.06 & 0.38 & -0.75 & -0.44 & 296 \\
\hline 195 & 12.33 & 12.80 & 0.36 & 0.48 & +0.01 & +0.12 & 3 \\
\hline 242 & 13.24 & 13.67 & 1.06 & 1.19 & +0.25 & +0.82 & 4 \\
\hline 245 & 11.57 & 12.25 & 0.18 & 0.76 & -0.54 & +0.25 & 293 \\
\hline 353 & 12.02 & 12.73 & 0.38 & 0.46 & +0.25 & +0.29 & 3 \\
\hline
\end{tabular}

\subsection{Spectroscopy}

Spectroscopic data of our programme stars were obtained during three observing runs, in June 1983, May 1985, and in June 1992, with the ESO $1.52 \mathrm{~m}$ telescope at the European Southern Observatory, La Silla, Chile. In 1983 and in 1985 the telescope was equipped with an Image Dissector Scanner (IDS) mounted at the Boller and Chivens spectrograph, whereas in 1992 the telescope was equipped with a Ford Aerospace 2048 CCD and an identical spectrograph. The IDS spectra were obtained through a $4^{\prime \prime} \times 4^{\prime \prime}$ aperture, whereas for the CCD long-slit spectra a slit with a width of $2^{\prime \prime}$ was used. During all observing runs a grating with a dispersion of $172 \AA \mathrm{mm}^{-1}$, centered at $5600 \AA$, was used. The 1983 and 1985 spectra were reduced using IHAP, whereas the 1992 spectra were reduced using MIDAS. All three sets of spectra were reduced at ESO Headquarters, Garching bei München, Germany. In order to obtain an optimal $\mathrm{S} / \mathrm{N}$ ratio for our spectra the optimal extraction algorithm by Horne (1986) was employed for the extraction of the 1992 spectral data from the CCD images. In the IDS spectra, sky subtraction was achieved by subtracting a simultaneously measured reference spectrum 2 arcminutes from the star, whereas for the CCD spectra sky subtraction was achieved by subtracting a third degree polynomial fitted to the spectrum 100 pixels (corresponding to roughly $25^{\prime \prime}$ ) in the spatial direction of the stellar spectrum. Because of the two-dimensional nature of the 1992 CCD long-slit spectra the results of the employed correction for the emission from surrounding nebulosity are clearly superior to the results of this correction in the 1983 and 1985 spectra. It should be noted here, however, that the sky subtraction in both cases sometimes leaves some residual emission, especially in $\mathrm{H} \alpha$, of which it is not clear whether this is real or if this is due to the surrounding nebulosity. Examples of the 1983 and 1985 IDS spectra are shown in Fig. 1. The absolute flux scales 

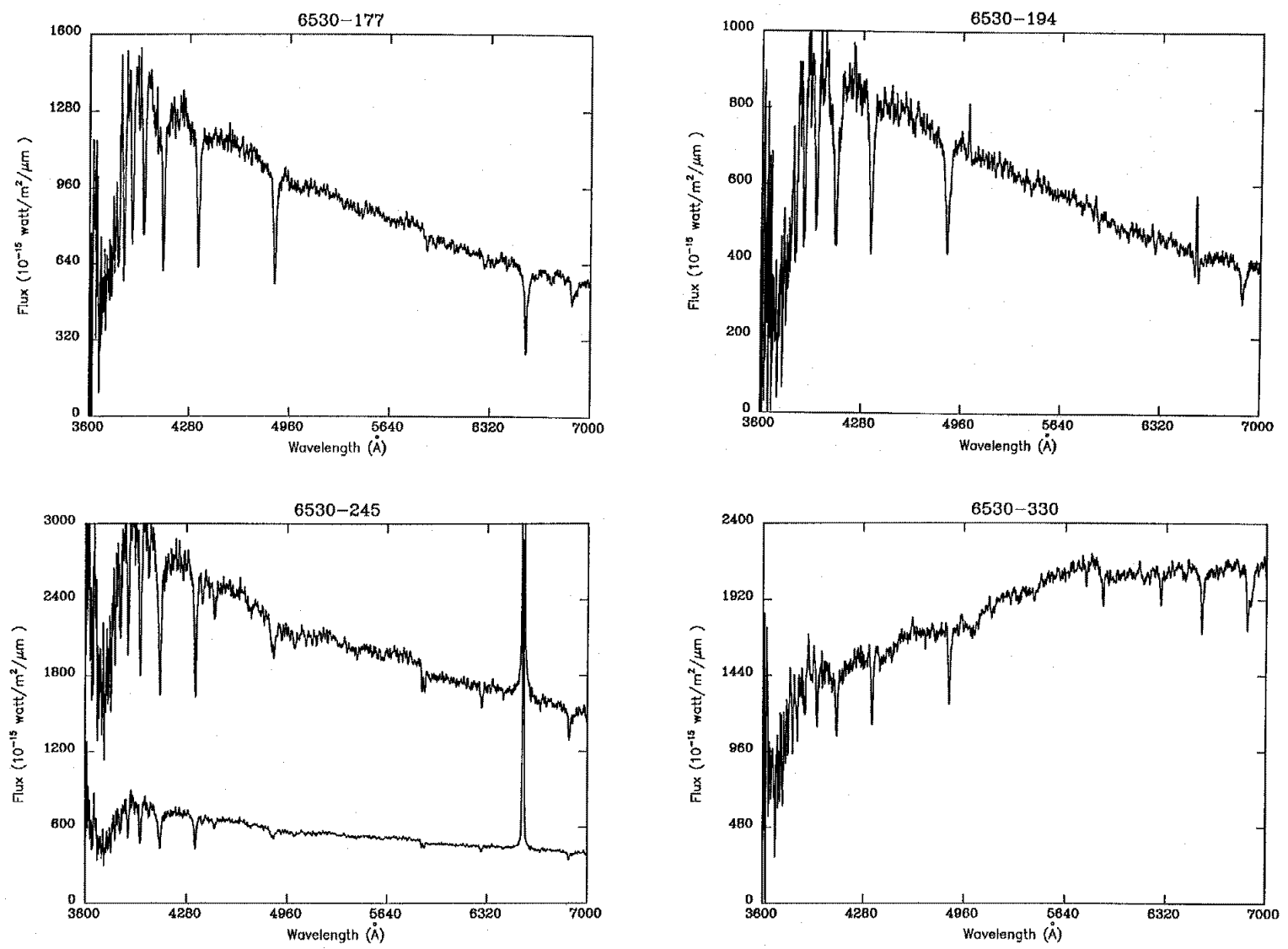

Fig. 1. Examples of spectra of programme stars in NGC 6530 taken with the ESO $1.5 \mathrm{~m}$ telescope with IDS detector. The top line in the plot for NGC 6530-245 shows an enlarged version of the spectrum

Table 4. Comparison of NGC 6530 photometric data sets with Chini \& Neckel (1981), with $\Delta V=\overline{V_{\mathrm{C} \& \mathrm{~N}}-V}$

\begin{tabular}{lrcrr}
\hline Author & $\Delta V$ & $\Delta(B-V)$ & $\Delta(U-B)$ & $n$ \\
\hline Walker (1957) & $0.006 \pm 0.006$ & $-0.027 \pm 0.005$ & $0.069 \pm 0.011$ & 49 \\
Kilambi (1977) & $0.018 \pm 0.017$ & $-0.027 \pm 0.016$ & $-0.017 \pm 0.024$ & 69 \\
Sagar \& Joshi (1978) & $-0.017 \pm 0.007$ & $-0.039 \pm 0.007$ & $0.093 \pm 0.020$ & 81 \\
Stal'bovskii \& Shevchenko (1981) & $-0.048 \pm 0.008$ & $0.002 \pm 0.011$ & $0.000 \pm 0.023$ & 6 \\
This paper & $-0.011 \pm 0.007$ & $-0.032 \pm 0.008$ & $0.039 \pm 0.016$ & 44 \\
This paper, Walraven data & $-0.039 \pm 0.011$ & - & - & 26 \\
\hline
\end{tabular}

in these figures compare within $0{ }^{\mathrm{m}} 10$ with the measured photometry. All 1992 CCD spectra are shown in Fig. 2.

New spectral classifications for our programme stars, in the MK system, were made by comparing the spectra with each other as well as with the standard MK spectra by Jacoby et al. (1984). The results of these classifications are listed in Table 5. The error in the resulting spectral types will generally be smaller than 2 subclasses, although for spectra with a poor $\mathrm{S} / \mathrm{N}$ ratio the errors may be much larger (indicated by a colon following the spectral type in Table 5). The stars in which some residual $\mathrm{H} \alpha$ emis- sion of unclear origin is seen are classified as e:. Whether these small emission components are due to intrinsic $\mathrm{H} \alpha$ emission or are due to imperfect sky subtraction remains unclear. The stars labelled with e certainly do possess intrinsic $\mathrm{H} \alpha$ emission, however.

\section{Derivation of stellar parameters}

For all programme stars we made additional spectral classifications from photometry by dereddening all programme stars in the $(U-B)$ versus $(B-V)$ 

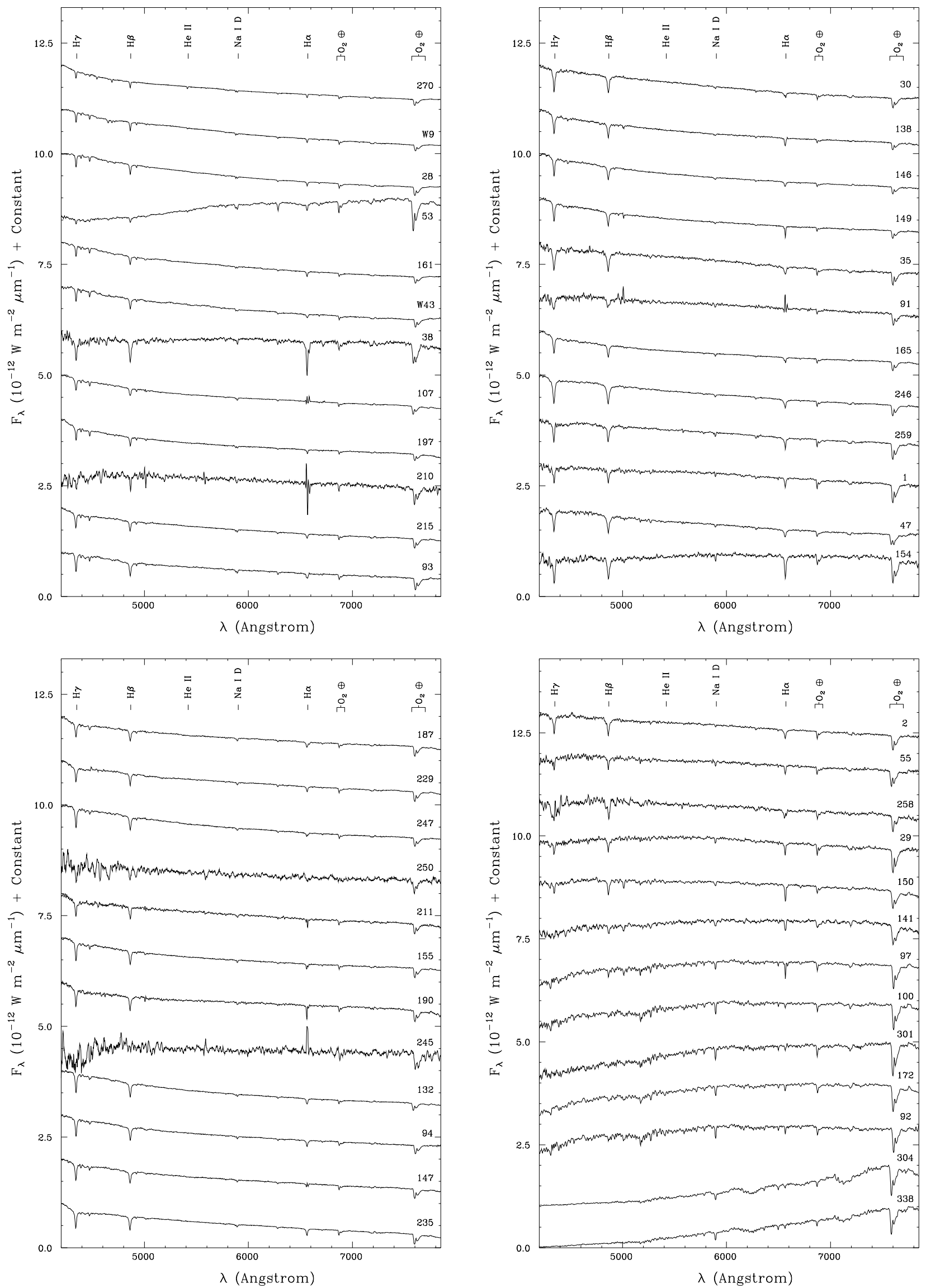

Fig. 2. Spectra of programme stars in NGC 6530 taken with the ESO $1.5 \mathrm{~m}$ telescope with CCD detector 
diagram (Fig. 3), assuming a normal extinction law, i.e. $E(U-B) / E(B-V)=0.72$. When available the new $U B V$ data were used in this diagram. In other cases we used the photometry by Chini \& Neckel (1981) or by Sagar \& Joshi (1981). As can be seen from Fig. 3 as well as from the colour-magnitude diagrams (shown in Fig. 4), it is not possible to distinguish giants from main-sequence stars using this $(U-B)$ versus $(B-V)$ diagram. Therefore we assumed all stars to be of luminosity class $\mathrm{V}$ in this classification. The resulting spectral classifications using $U B V$ data from literature are listed in the second column of Table 5 , whereas the resulting classifications using the new $U B V$ data from Table 2 are listed in the third column. A dash in these columns indicates that $U B V$ data was available, but the corresponding reddening line did not intersect the main sequence.

For the stars with photometric data in the Walraven system we calculated the reddening-free parameters $[B-U],[U-W]$ and $[B-L]$, defined as:

$[B-U]=(B-U)-0.61 \times(V-B)$

$[U-W]=(U-W)-0.45 \times(V-B)$

$[B-L]=(B-L)-0.39 \times(V-B)$.

In this method the extinction law is again assumed to be normal. The resulting $[B-U]$ versus $[B-L]$ and $[B-U]$ versus $[U-W]$ diagrams are shown in Fig. 5. In these diagrams we also plotted lines of constant $T_{\text {eff }}$ and $\log g$, derived from Kurucz (1991) models in a similar way as was done for the Kurucz (1979) models by Brand $\&$ Wouterloot (1988). Our new $[B-U]$ versus $[B-L]$ diagram agrees well with theirs, whereas clear differences can be seen between the $[B-U]$ versus $[U-W]$ diagrams. As can be seen from Fig. 5, the data points fall reasonably well within the grid of Kurucz models for the $[B-U]$ versus $[B-L]$ diagram, whereas for the $[B-U]$ versus $[U-W]$ diagram a fair amount of data points are plotted outside the grid. This same effect was also noted by Brand \& Wouterloot (1988), who attributed this to the failure of the Kurucz (1979) models to reproduce the absorption line strengths in the $W$ band. We conclude that although the Kurucz (1991) models do give better results than the ones from 1979, this still is the case. Following Brand \& Wouterloot (1988), we only used the $[B-U]$ versus $[B-L]$ diagram (Fig. 5b) to derive a two-dimensional spectral type by obtaining values for $T_{\text {eff }}$ and $\log g$ by interpolation between the lines of constant $T_{\text {eff }}$ and $\log g$, and converting these to two-dimensional spectral types, adopting the absolute calibrations by Schmidt-Kaler (1982). The resulting spectral classifications are listed in the fourth column of Table 5. As can be seen from Fig. 5b a small number of points fall outside the scope of the grid covered by the Kurucz models in the $[B-U]$ versus $[B-L]$ diagram as well. The programme stars for which this is the case are indicated in Table 5 by dashes in the fourth column.
Deviations in both the $[B-U]$ and $[B-L]$ directions may be due to the assumption of a normal extinction law in deriving the reddening-free parameters $[B-U]$ and $[B-L]$. Since the Walraven $L$ band is heavily influenced by the Balmer lines in this band, the deviations in the $[B-L]$ direction may very well be explained by the presence of hydrogen emission lines in the stellar spectrum, either intrinsic or due to poor sky subtraction. In retrospective, it is of course not surprising that the unsophisticated sky subtraction procedure offered by our aperture photometry often fails, for bands heavily influenced by hydrogen emission lines, when even the sophisticated sky subtraction procedures used for the reduction of our CCD long-slit spectra are probably not always successful in removing all emission from the surrounding nebulosity.

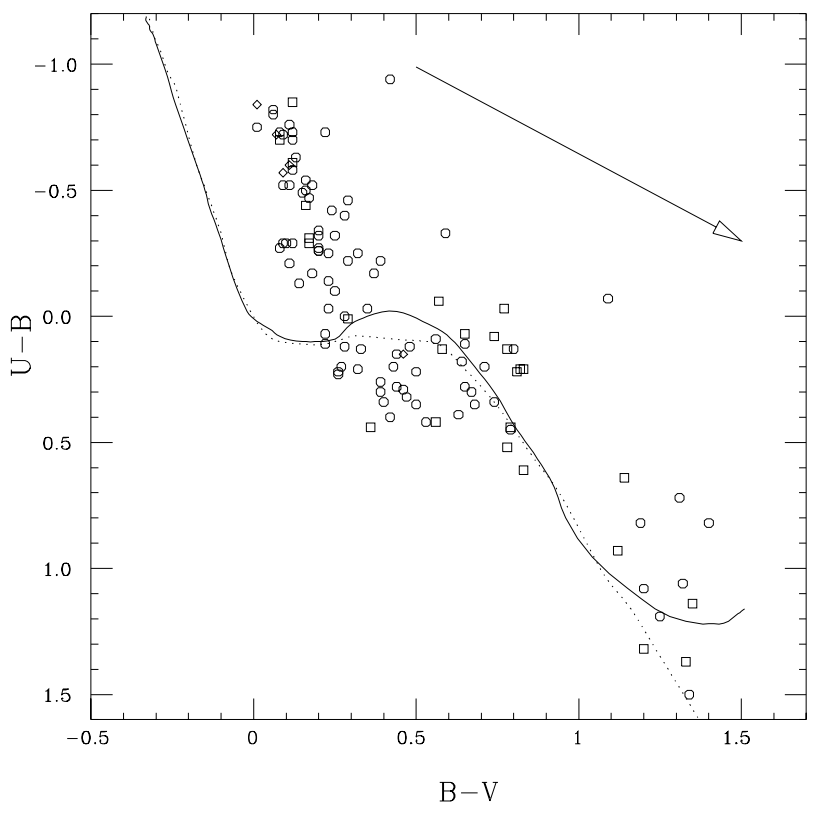

Fig. 3. The $U-B$ versus $B-V$ two colour diagram for programme stars in NGC 6530. Probable members $(P \geq 50 \%)$ are indicated by circles. Possible members $(10 \% \leq P<50 \%)$ are indicated by squares. The diamonds indicate programme stars not included in the proper motion survey by van Altena \& Jones (1972). The solid line shows the intrinsic colours for main-sequence stars (luminosity class V). Those for luminosity class III (giants) are indicated by the dashed line. The arrow indicates the reddening direction for a normal extinction law, with $E(U-B) / E(B-V)=0.72$

In comparing the spectral types listed in Table 5 found using the different methods we notice that generally there is good agreement between the spectral types obtained from spectroscopy and photometry, indicating that the assumption of a normal extinction law in obtaining the spectral types from photometry was not too far off. In individual cases we can notice large differences between 
the spectroscopic and photometric (especially from the Walraven data) spectral types, however. This could e.g. be due to the presence of unresolved binaries. In such cases we adopted the spectroscopic spectral type for the programme stars if this was well-determined. In all other cases we took the spectral types from the Walraven or Johnson photometry, when available and in agreement with the rough spectroscopic spectral types. These adopted spectral types are listed in the $7^{\text {th }}$ column of Table 5 . Colour excesses $E(B-V)$ were determined from the observed $(B-V)$, adopted spectral types, and the intrinsic $(B-V)$ colours from Schmidt-Kaler (1982) and are also listed in Table 5.

Spectral Energy Distributions (SEDs), from the ultraviolet to the infrared wavelength ranges, were constructed for all programme stars using the new photometric data assembled in Table 2, photometric data from literature and ultraviolet spectrophotometric data extracted from the IUE archives. Observed magnitudes/intensities were converted to fluxes using the flux calibrations by Wesselius et al. (1982), de Ruiter \& Lub (1986), Johnson (1966), Bessell (1979) and Berrilli et al. (1992) for the ANS, Walraven, Johnson, Cousins and Near-IR data, respectively.

These observed SEDs were corrected for interstellar plus circumstellar extinction by comparing the observed fluxes $\left(F^{\text {obs }}\right)$ to the theoretically observed fluxes $\left(F^{\text {th }}\right)$ at earth:

$F^{\mathrm{th}}=\int_{0}^{\infty}\left(\frac{\pi R_{\star}^{2}}{d^{2}} F_{\lambda} \operatorname{dex}\left(-\frac{A_{\lambda}}{2.5}\right)\right) S_{\lambda} \mathrm{d} \lambda$.

In this formula $F_{\lambda}$ is the flux emitted by the star per surface unit, $R_{\star}$ is the stellar radius, $d$ is the stellar distance, $A_{\lambda}$ is the total extinction at a given wavelength (in magnitudes) and $S_{\lambda}$ is the transmission function of the photometric filter. $F_{\lambda}$ was taken from the Kurucz (1991) models for the adopted spectral type, assuming a solar abundance of the elements. $S_{\lambda}$ was taken from SchmidtKaler (1982) for the Walraven, Johnson and Cousins photometric systems, from Wesselius et al. (1982) for the ANS data and from Fluks et al. (1996) for the Near-IR data, after which we normalized these transmission functions so that $\int_{0}^{\infty} S_{\lambda} \mathrm{d} \lambda=1 . A_{\lambda}$ (as a function of the ratio of total to selective extinction $R_{V}$ ) was obtained from the extinction laws by Steenman \& Thé (1991), using the relation $A_{V}=R_{V} \times E(B-V)$. Since $R_{\star}$ and $d$ are not accurately known, the parameters $\pi R_{\star}^{2} / d^{2}$ were obtained by fitting $F^{\text {obs }}$ to $F^{\text {th }}$ in each SED. This fitting procedure was applied only up to $1.3 \mu \mathrm{m}$ ( $J$-pass band), because beyond this wavelength the SED could be influenced by thermal emission of circumstellar dust grains in which case the Kurucz model does not represent the true system flux anymore. Examples of the resulting observed and extinctionfree SEDs are shown in Fig. 6.

With this method it is also possible to correct for anomalous extinction, characterized by a value of $R_{V}$, by fitting $F^{\text {obs }}$ to $F^{\text {th }}$ for different values of $R_{V}$ using the method described above, until, by trial and error, a best fit is obtained, according to the $\chi^{2}$-test. If a value significantly different from the one found for interstellar matter, $R_{V}=3.1$, is found the extinction law is called anomalous, which indicates a different size distribution or chemical composition of the dust grains responsible for the extinction than those in interstellar matter. If the extinction is anomalous $\left(R_{V}>3.1\right)$, the error in the $R_{V}$-value is 0.2 , whereas for $R_{V}=3.1$ this error is 0.1 , provided that data points over a sufficient wavelength interval were used. Note that the obtained extinction laws are in fact a mixture of an interstellar, normal, and a possible circumstellar, potentially anomalous, component. So, in the case of anomalous extinction the $R_{V}$-values for the circumstellar matter will in fact be higher than the ones derived using this method.

Note that above procedure to analyze the SED is very similar to the one employed by Steenman \& Thé (1989), but has been improved by the use of the new extinction laws by Steenman \& Thé (1991), and the use of the new Kurucz (1991) models. Furthermore, we used fluxes integrated over the response curve of the photometric band, instead of monochromatic fluxes.

For most programme stars we obtained good fits of the extinction-corrected fluxes to the Kurucz model. However, there are a number of stars in which the $J H K$ photometry lies above the Kurucz model, and thus exhibit infrared excesses, probably due to remnants of a circumstellar disk or dust shell left over from star formation. The stars for which this is the case are marked with "Yes" in the last column of Table 5, whereas for stars in which we obtain a good fit of the $J H K$ bands to the Kurucz model, this column contains "No". The dashes in this column indicate that not enough data were available to draw any definite conclusions. It is unlikely that these infrared excesses are due to the presence of red companions because: (a) the shape of most of these infrared excesses are not compatible with stellar colours; (b) unperturbed late-type stars will not have had time to loose enough circumstellar material to become even visible in the near infrared (Palla \& Stahler 1993), so it is very unlikely that such an infrared excess is due to optical binaries; (c) in case an early type star has a late-type companion, it sweeps out its circumstellar environment very rapidly (e.g. Hester et al. 1996; Pérez et al. 1996), after which the difference in brightness between the primary and the secondary will be so large that this would not show up in our study. Furthermore, if an infrared excess would be due to a late-type companion, we would expect that it would be bright enough to detect its presence in our spectra. 

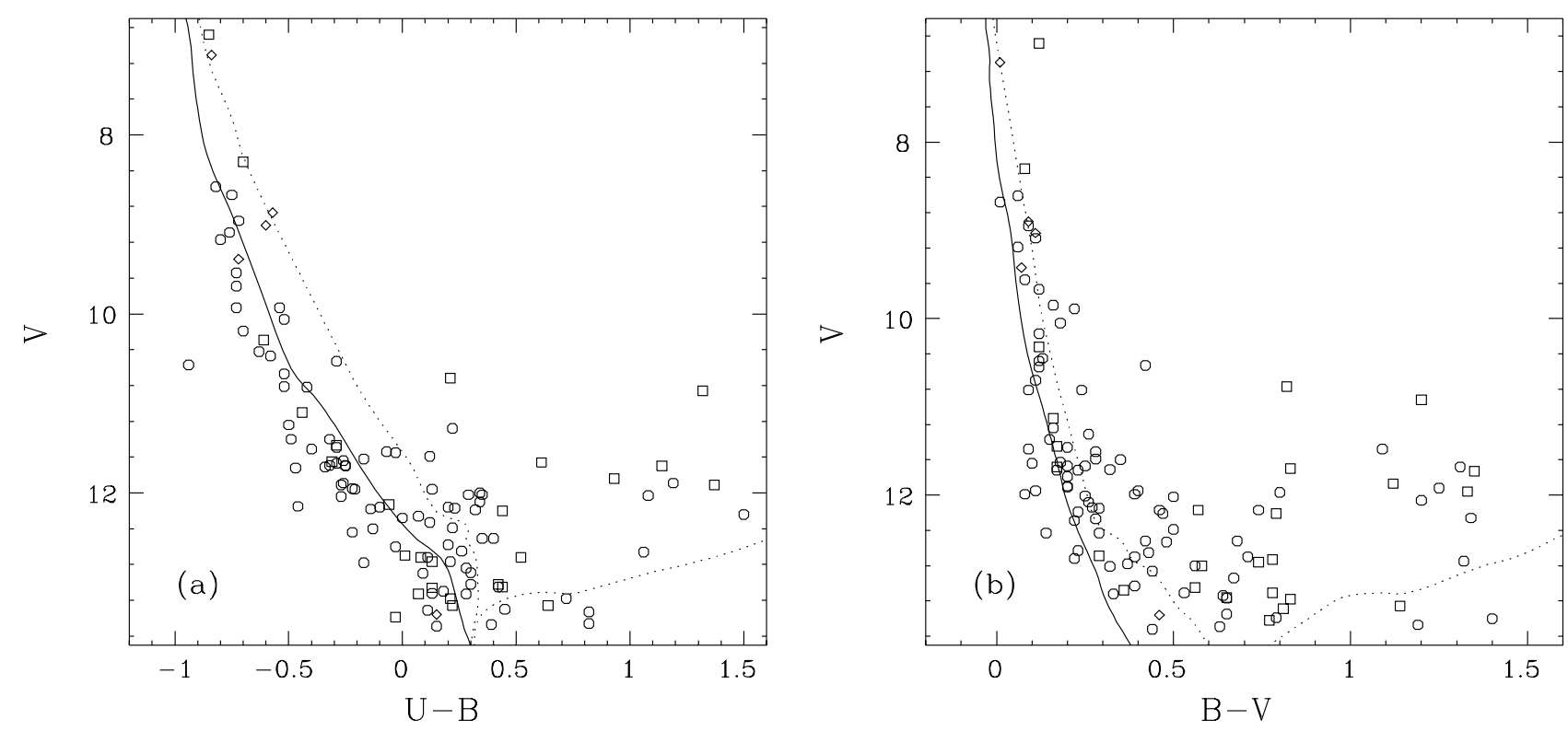

Fig. 4. a) $V$ versus $(U-B)$ colour-magnitude diagram for programme stars in NGC 6530. Plot symbols have the same meaning as in Fig. 3. The solid line shows the colour-magnitude relation for stars of luminosity class $\mathrm{V}$ with a distance modulus of 11 . 28 and corrected for reddening and extinction with $E(B-V)=0.30$ (see Sect. 4). The relation for luminosity class III (giants) is indicated by the dashed line. b) $V$ versus $(B-V)$ colour-magnitude diagram for programme stars in NGC 6530 . Plot symbols and lines have the same meaning as in a)
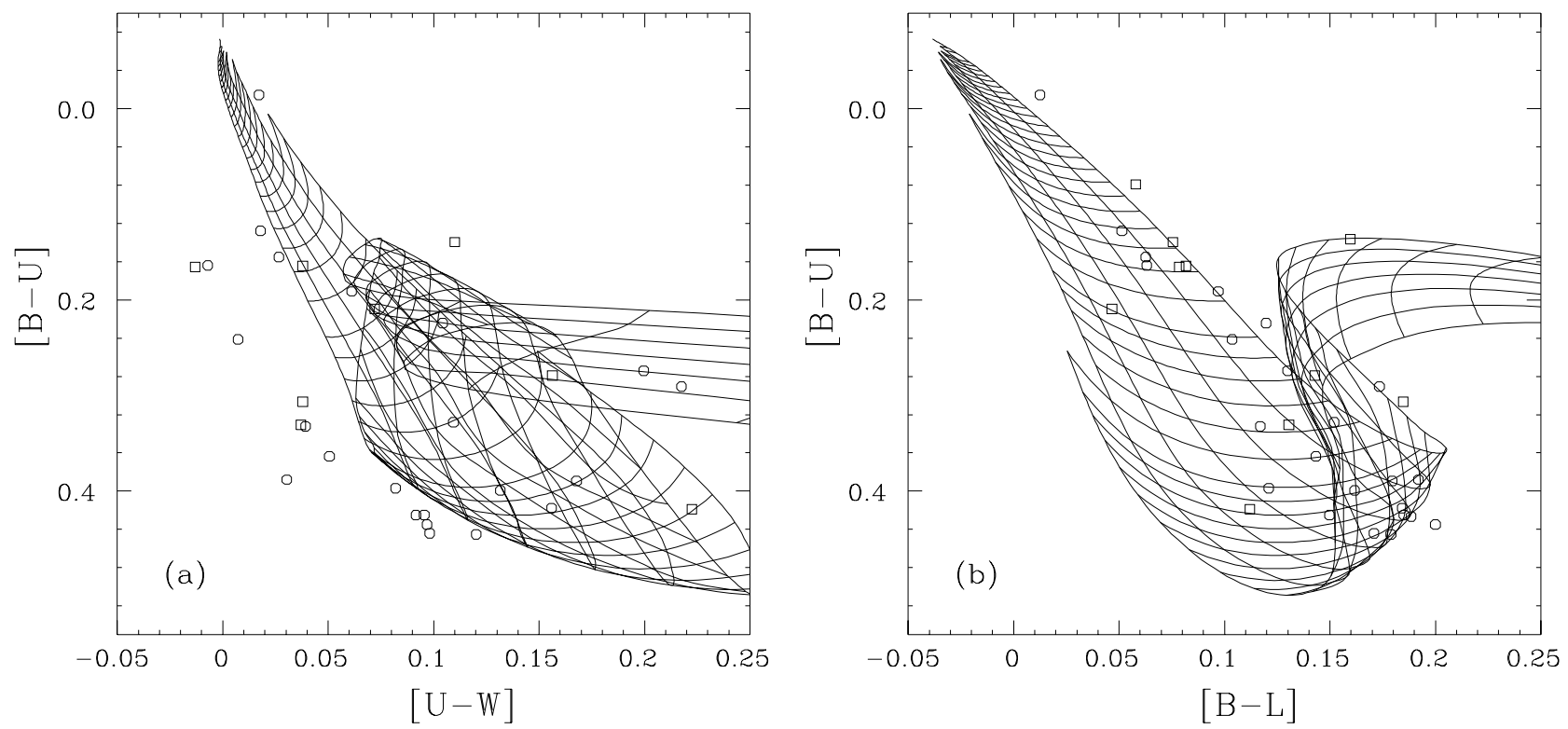

Fig. 5. a) The Walraven $[B-U]$ versus $[U-W]$ two colour diagram for our programme stars in NGC 6530 with Walraven photometric data. Probable members $(P \geq 50 \%)$ are again indicated by circles, whereas possible members $(10 \% \leq P<50 \%)$ are again indicated by squares. Lines of constant $T_{\text {eff }}$ and $\log g$, according to the Kurucz (1991) models, are also shown. b) The Walraven $[B-U]$ versus $[B-L]$ two colour diagram for our programme stars in NGC 6530 with Walraven photometric data. Plot symbols and lines have the same meaning as in a) 


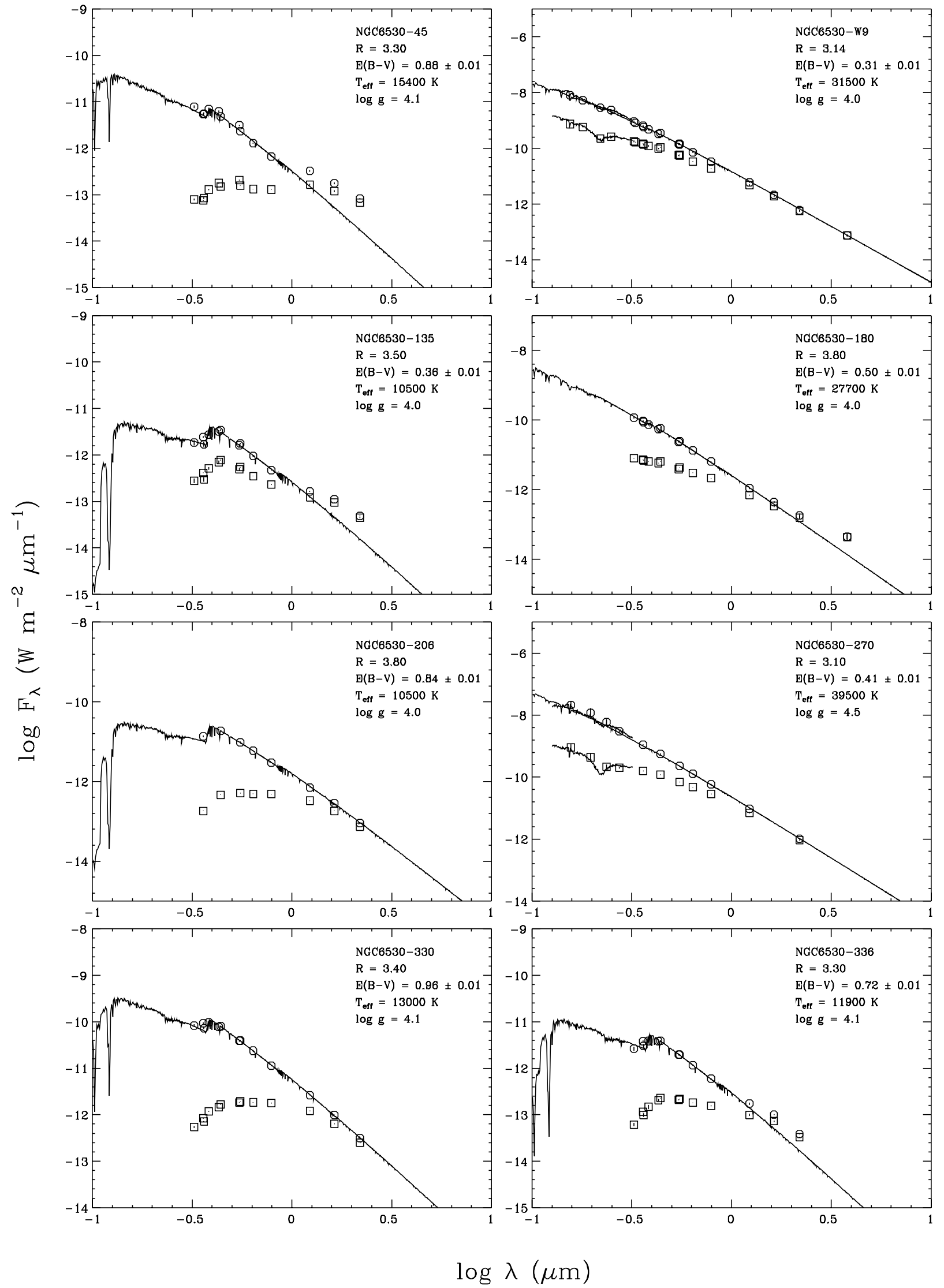

Fig. 6. Examples of observed (squares) and extinction-free (circles) SEDs for our programme stars. The solid line is the Kurucz (1991) model fitted through the extinction-free SED 
After applying our SED-fit method, stellar luminosities were computed by integrating over the Kurucz (1991) model, fitted to the extinction-free SED:

$\frac{L_{\star}}{d^{2}}=4 \pi \int_{0}^{\infty} F_{\lambda} \mathrm{d} \lambda$,

with $F_{\lambda}$ the Kurucz model flux, fitted to the extinctioncorrected SED. For wavelengths longer than the largest one covered by the Kurucz (1991) model $(200 \mu \mathrm{m}), F_{\lambda}$ was taken equal to a Planckian in the Rayleigh-Jeans limit, fitted to the Kurucz model, and the analytical solution of the resulting integral was used to perform the integration to infinity. A distance $d_{\text {phot }}$ was derived for each programme star by comparing this stellar luminosity with the absolute luminosity calibration assembled by Schmidt-Kaler (1982). The above method to derive distances of individual objects gives similar results as the more familiar distance modulus method. However, our method will give more accurate results because it does not rely on the accuracy of only one photometric measurement (mostly $V$ ) and on often poorly estimated bolometric corrections. The computed values of $E(B-V), R_{V}$ and $d_{\text {phot }}$, as well as $\log T_{\text {eff }}$ and $\log L_{\star} / L_{\odot}$, adopting a distance of $1.8 \pm 0.2 \mathrm{kpc}$ (see Sect. 4), for each star are listed in Table 5. A dash in the column with $R_{V}$ values or the infrared excess indicates that not enough data was available to draw any definite conclusions on this. A colon indicates uncertain values.

\section{Cluster properties}

\subsection{Extinction}

Using the data in Table 5, we constructed a histogram with the distribution of the colour excess $E(B-V)$, shown in Fig. 7. As can be seen from this figure as well as directly from the $B-V$ versus $U-B$ two-colour diagram (Fig. 3), most stars are located in a strip with $E(B-V)$ from 0.34 to 0.40 . Therefore, we conclude that the foreground interstellar reddening is probably about 0.30 , and stars with a smaller colour excess must be foreground objects. Since the error in most $E(B-V)$ 's is about $0{ }^{\mathrm{m}} 02$, all stars with $E(B-V)<0.28$ were excluded from the further analysis of cluster properties. Another interesting feature that can be seen in Fig. 7 is that there also seem to be peaks around $E(B-V)=0.54$ and $E(B-V)=0 \mathrm{~m} 92$. This could indicate the existence of clouds of obscuring material in NGC 6530 . To test this hypothesis we plotted the positions of the programme stars with $0.28 \leq E(B-V)<00^{\mathrm{m}} 41$, as well as the positions of stars with $0.42 \leq E(B-V) \leq 0{ }^{\mathrm{m}} 64$ and with $0^{\mathrm{m}} \cdot 66 \leq E(B-V) \leq 1^{\mathrm{m}} \cdot 12$. In these three diagrams no significant differences could be found in the distribution of the stellar positions. Therefore we conclude that if these peaks around $E(B-V)=0.54$ and $E(B-V)=0.92$ are really due to clouds of obscuring material in NGC 6530, these clouds must span the entire field of the cluster. It seems more likely that these peaks really are stochastic noise, however. Furthermore, we conclude that we could not reproduce the variable interstellar reddening across NGC 6530 that was observed by Sagar \& Joshi (1978). We believe that the difference between our and their conclusion is the result of a bigger sample and better selection criteria for probable cluster members in our study.



Fig. 7. Histogram of the distribution of colour excesses $E(B-V)$ in NGC 6530

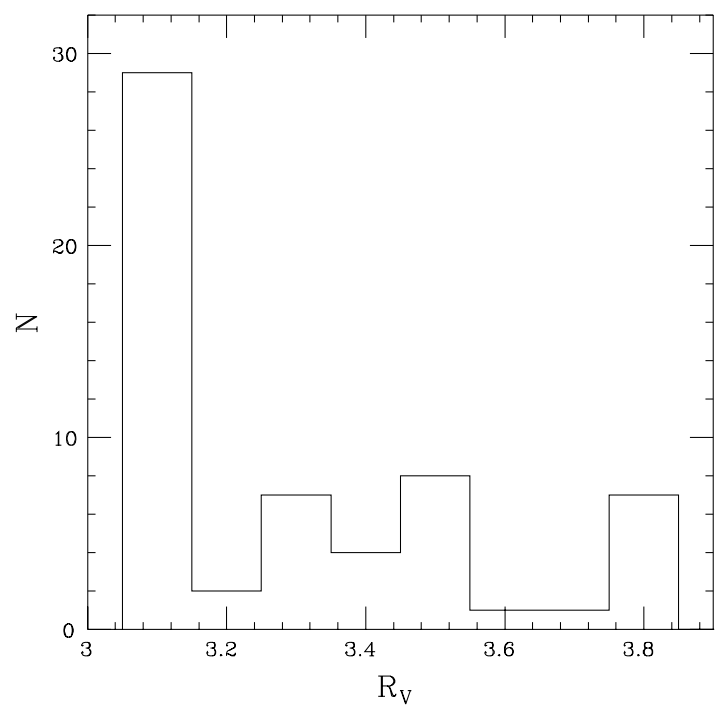

Fig. 8. Histogram of the distribution of $R_{V}$-values in NGC 6530

Again using the data in Table 5, we also constructed a histogram with the distribution of the ratios of total to 
selective extinction $R_{V}$, shown in Fig. 8. As can be seen from this figure, most stars in NGC 6530 do not show anomalous extinction (i.e. $R_{V} \neq 3.1$ ), and none show extreme values of $R_{V}$, a situation similar to that found in very young open clusters like NGC 6193 (Vázquez \& Feinstein 1992). There are clearly some cases of anomalous extinction with $R_{V}>3.2$ (i.e. the average particle size of the material in our line of sight is larger than that in the interstellar medium), but all of these also have values of $E(B-V) \gg 0$ m 30 . However, most stars with these big values of $E(B-V)$ do not show anomalous extinction. From this we conclude that the matter responsible for the anomalous extinction must be circumstellar rather than intracluster. This also means that each star has its own individual extinction law, and that the derivation of an average extinction law for a very young open cluster, such as was done by McCall et al. (1990) for NGC 6530, will not yield correct results.

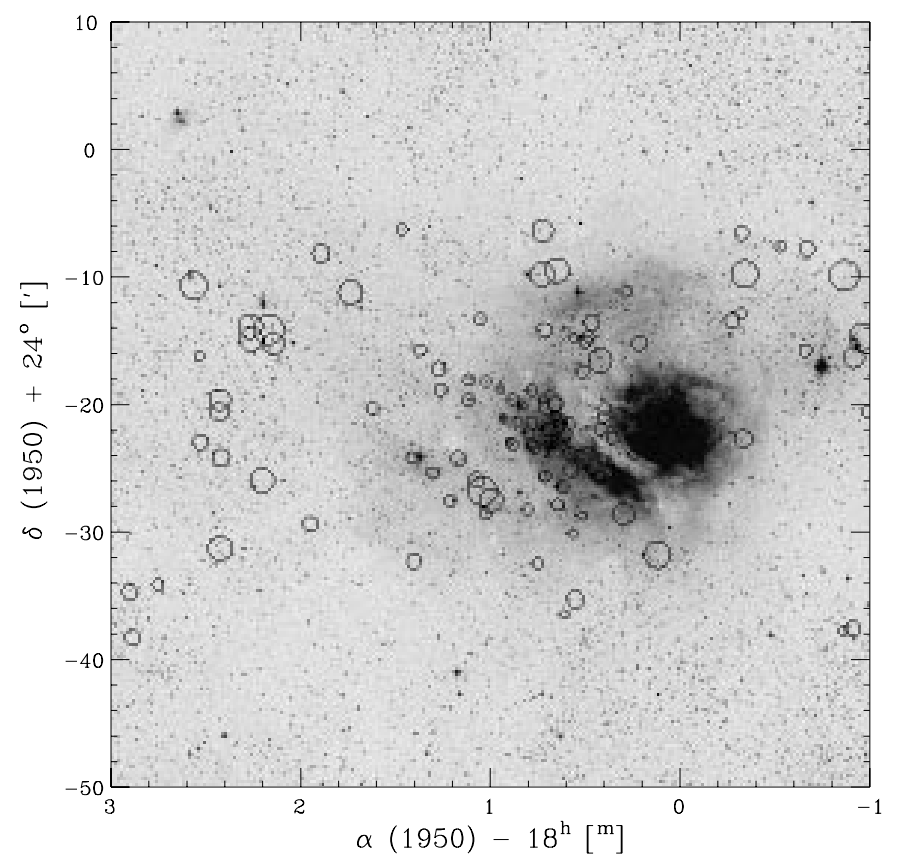

Fig. 9. The field of NGC 6530 with the position of our programme stars indicated by circles. The size of the circle indicates the magnitude of the visual extinction $A_{V}$. Again stars with $E(B-V)<0.28$ (i.e. probable foreground objects) were omitted

Combining the $R_{V}$ and $E(B-V)$ data from Table 5 we also constructed a diagram with the visual extinction $A_{V}=R_{V} \times E(B-V)$ as a function of the positions in the cluster, shown in Fig. 9. Also shown in this figure is the cluster field as obtained from the digitized version of the Palomar Observatory Sky Survey (POSS). From Fig. 9 we notice that bigger values of $A_{V}$ (indicated by larger circles) seem to occur more often in the outer parts of the cluster, whereas these large values of $A_{V}$ seem to be quite rare in the inner, more nebulous, part of NGC 6530. This could indicate the presence of an obscuring dark cloud in the wider region of the cluster. However, no obvious dark cloud region could be found by visual inspection of POSS plates. Therefore we conclude that the larger $A_{V}$ values in the outer parts are probably due to contamination of our sample with background objects in the outer parts of NGC 6530, whereas in the inner parts most background stars are obscured by the bright nebulosity. Besides this effect, we do not note any particular correlation of the visual extinction with the position in the cluster, again indicating that the material responsible for the extra extinction must be circumstellar rather than intracluster.

\subsection{Distance}

A histogram with the distribution of the distances of individual stars in NGC 6530, obtained in the previous section by comparing the luminosity computed from the SEDs with the intrinsic ones collected by Schmidt-Kaler (1982), is shown in Fig. 10. A gaussian was fitted to this distribution, yielding an average distance of $1.8 \pm 0.2 \mathrm{kpc}$ towards NGC 6530, in excellent agreement with the value of $1.86 \pm 0.07 \mathrm{kpc}$ found by McCall et al. (1990).

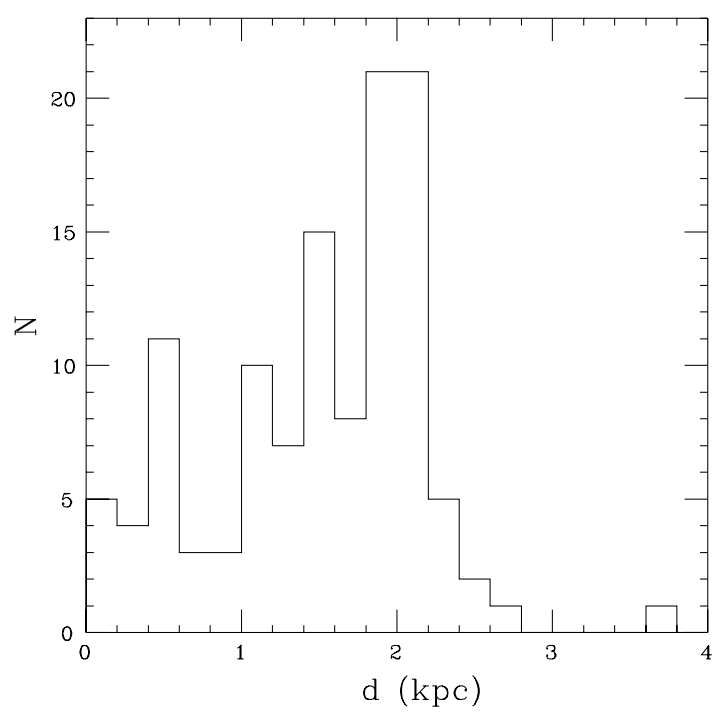

Fig. 10. Histogram of the distribution of the derived distances to individual programme stars in NGC 6530

At this distance the cluster diameter of roughly 35 arcminutes corresponds to $18 \mathrm{pc}$, or two to three time bigger than very young open clusters like NGC 2244, NGC 2264 and NGC 6383 (Pérez et al. 1987; Thé et al. $1985)$. However, this $18 \mathrm{pc}$ is comparable to the diameter of NGC 6611 (de Winter et al. 1996). Remarkable is that although we expect the total volume of NGC 6530 or 
NGC 6611 to be about 40 times bigger than NGC 2244, NGC 2264 or NGC 6383 , the number of OB stars in NGC 6530 (90) is only a factor of three bigger and in NGC 6611 even comparable to that in these clusters. Whether this lower spatial density of hot stars in NGC 6530 and NGC 6611 is due to evolutionary effects or is the result of different initial conditions remains unclear at the moment.

\subsection{HR-diagram}

Using the distance obtained in the last $\S$ and the computed values for $L_{\star} /\left(d^{2} L_{\odot}\right)$, we constructed the cluster's Hertzsprung-Russell diagram, shown in Fig. 11. For most points in this plot the error in $\log T_{\text {eff }}$ will be about 0.05 (or one subclass in spectral type), but individual data points may have larger errors. The error in $\log L_{\star} / L_{\odot}$ is dominated by the error in the distance and is about 0.1 . The relative error with respect to other data points will be much smaller, though. Also shown in Fig. 11 are the premain sequence evolutionary tracks and the birthlines (i.e. the line where a star first becomes optically visible on its evolution to the main-sequence) for accretion rates of $10^{-4}$ and $10^{-5} M_{\odot} \mathrm{yr}^{-1}$ computed by Palla \& Stahler (1993). Furthermore, we also plotted a line indicating the completeness limit of our study. This was computed by fitting reddened (with a colour excess $E(B-V)$ of 0.30 ) Kurucz (1991) models to the limiting visual magnitude of $13{ }^{\mathrm{m}} 6$ from the proper motion study by van Altena \& Jones (1972), after which their luminosity was computed using formula (6). The stars which are located far below this line are probably foreground stars.

Remarkable is that we don't see any evidence for a horizontal branch of pre-main sequence stars in the HR-diagram like the one observed by Walker (1957). Therefore, his age determination of a few million years might very well be incorrect. Furthermore, we note that several stars in our HR-diagram are located to the right of the birthline. This situation is very similar to the one in the very young open cluster NGC 6611 (de Winter et al. 1996), which was explained by demonstrating that this cluster contains a mixture of normal main-sequence stars, young stars still contracting towards the main sequence as well as older postmain sequence stars evolving off the main sequence. In the next section we will demonstrate that this is also the case for NGC 6530. Since the oldest stars which are without any doubt associated with NGC 6530 are about 15 million years old and the youngest stars must be younger than 100000 years (see next section) we conclude that star formation in this cluster must have started a few times $10^{7}$ years ago and probably is continuing up to the present day. In view of the small amount of massive (heavier than 8 solar masses) stars located near the zero-age main-sequence in Fig. 11, we conclude that the formation of such stars must have stopped already, while the formation of lighter stars is still going on. There is no evidence for a conclusion that the massive stars were the first to form, however: older low-mass stars may also be present.

In Fig. 11 we also notice that the stars with infrared excesses (filled plot symbols) are all located close to the main sequence, whereas those without are scattered throughout the diagram. If these infrared excesses are due to the remnants of dust shells or circumstellar disks left over from star formation, we would expect that all of our infrared excess stars would also be the youngest and lie more towards the birthlines in our HR-diagram. As we observe quite the opposite, we conclude that besides age several other factors must determine the magnitude of the infrared excess, presumably corresponding to the survival time of a circumstellar disk or dust shell, in this very young open cluster. However, we only looked at the infrared excess at near-IR wavelengths. It is quite possible that many of the stars we classified as not having an infrared excess do show such an excess at mid- and far-IR wavelengths. This will not affect our conclusions, as the magnitude of these mid- and far-IR excesses will always be smaller than the infrared excesses we have found here.

Another interesting thing to notice is that all five stars in NGC 6530 showing intrinsic $\mathrm{H} \alpha$ emission in their spectra all have strong near-IR excesses, and are thus good candidates for members of the Herbig Ae/Be stellar group, and are located close to the main sequence. The stars right of the main sequence in this diagram, with $\log T_{\text {eff }}<3.75$, are most probably background giants. This will be discussed further in the next section.

\subsection{Luminosity function}

Again using the data from Table 5 we also constructed the luminosity function of NGC 6530 by binning over $0.2 \log L_{\odot}$ steps, shown in Fig. 12a. In order to get an idea of the effects of the rebinning of our (small) sample we also constructed the incremental luminosity function, shown in Fig. 12b. Also shown in Fig. 12 is the theoretical luminosity function for a cluster with an age of $310^{6}$ years by Fletcher \& Stahler (1994). Although perhaps some depletion of massive stars in NGC 6530 with respect to these theoretical models can be seen, we conclude that in general the two curves match adequately. The agreement between the theoretical and the observed curve is $69 \%$, according to the Kolmogorov-Smirnov test. In this computation data with $\log L_{\star} / L_{\odot}<2.1$ (the point where our completeness limit in the HR diagram intersects the birthline for an accretion rate of $10^{-5} M_{\odot} \mathrm{yr}^{-1}$ ) were omitted because of the incompleteness of our sample in that region. Also note that for luminosity functions with other ages we can also obtain satisfactory fits, demonstrating the difficulty in the observational tests of such models. 


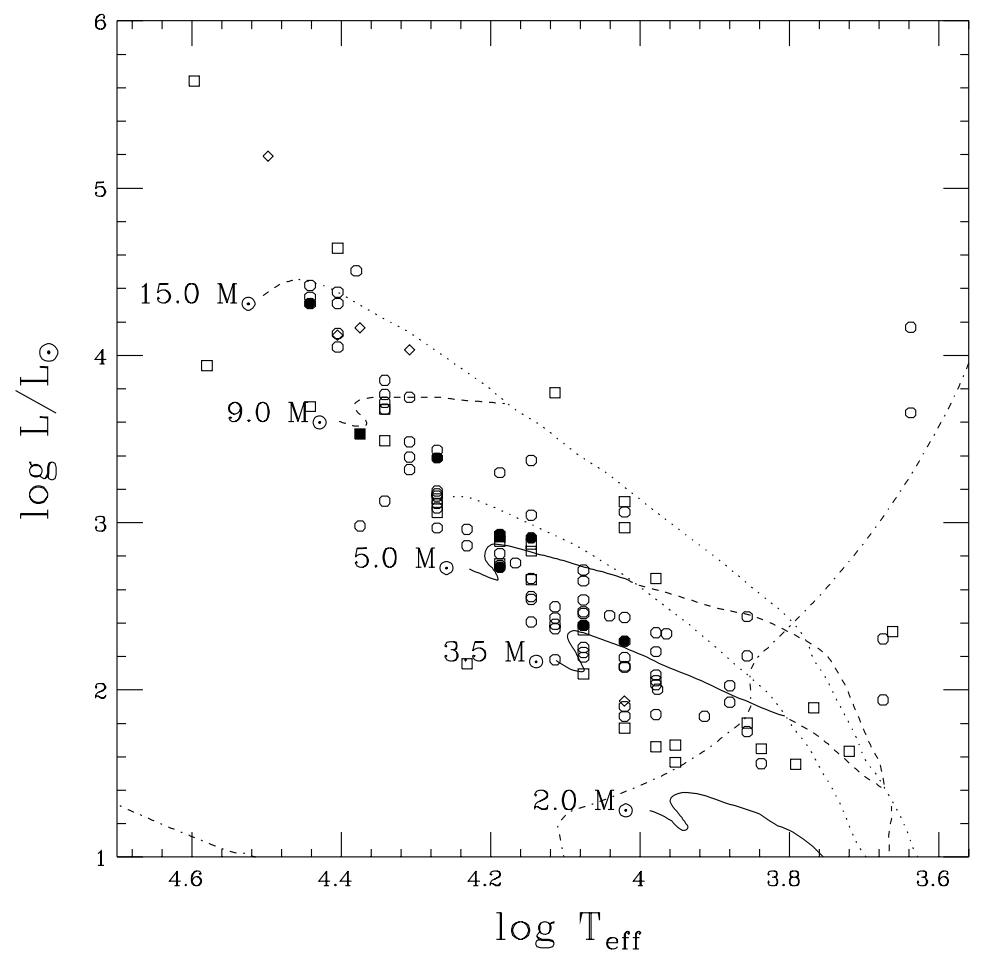

Fig. 11. Hertzsprung-Russell diagram of our programme stars in NGC 6530. Probable members $(P \geq 50 \%)$ are indicated by circles. Possible members $(10 \% \leq P<50 \%)$ are indicated by squares. The diamonds indicate programme stars not included in the proper motion survey by van Altena \& Jones (1972). Filled plot symbols indicate stars with infrared excess. Also shown are the theoretical pre-main sequence evolutionary tracks (solid lines and dashed lines) and the birthlines for $10^{-4}$ (upper dotted line) and $10^{-5} M_{\odot} \mathrm{yr}^{-1}$ (lower dotted line) by Palla \& Stahler (1993). The dashed-dotted line shows the completeness limit of our study
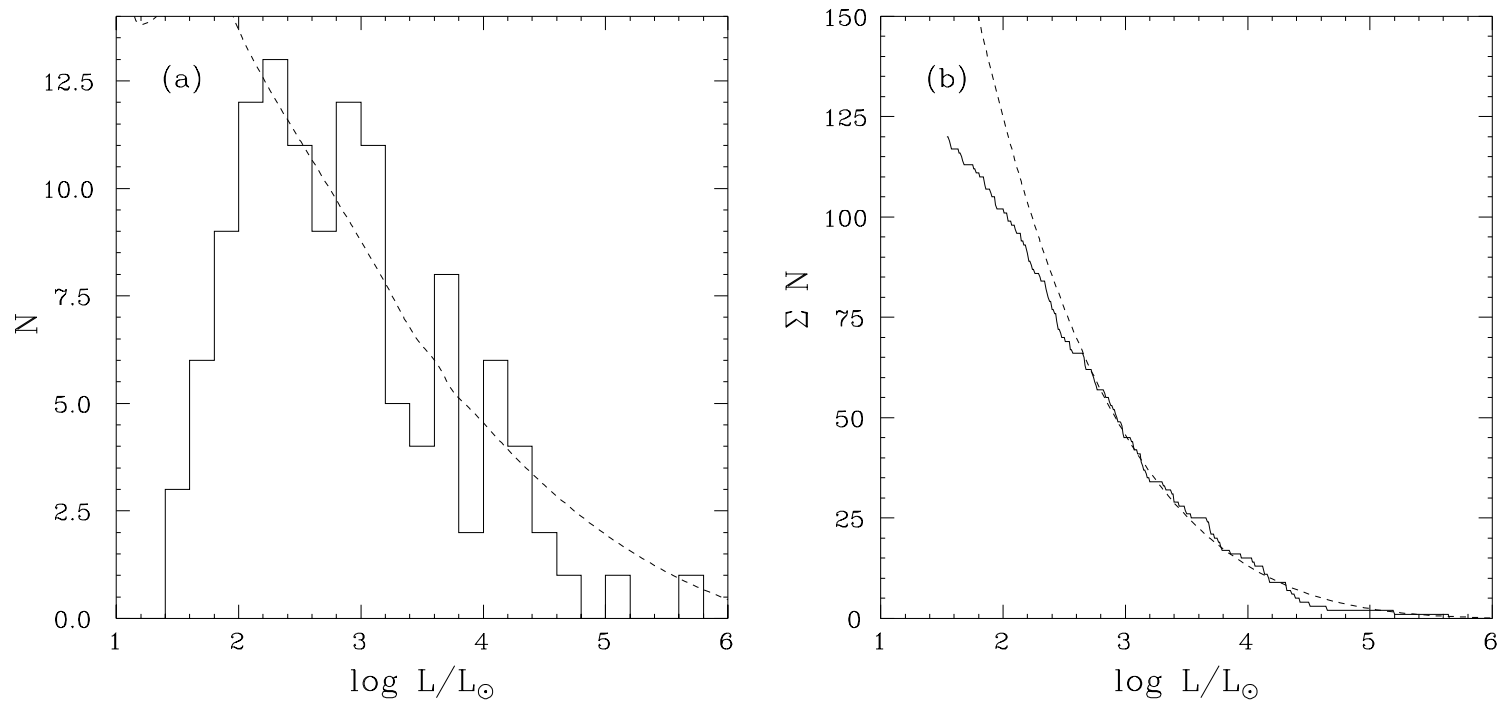

Fig. 12. Luminosity function of NGC 6530 (solid line) compared to the theoretical one for a cluster of 3 million years by Fletcher \& Stahler (1994) (dashed line). a) Luminosity function binned in $0.2 \log L_{\odot}$ steps. b) Incremental luminosity function 


\section{Notes on individual objects}

45. This star shows large $(>1.5)$ photometric variations, as well as probably some $\mathrm{H} \alpha$ emission and a strong infrared excess. In the Hertzsprung-Russell diagram (HRD) it is located close to the main sequence, near the pre-main sequence evolutionary track of a $5.5 M_{\odot}$ star, confirming its membership to NGC 6530 . We conclude that this cluster member is a good new Herbig Be candidate, which is near the end of its pre-main sequence phase.

53. Although in our CCD spectrum we recognize the spectral characteristics of an early B-type star, the slope of this spectrum seems to suggest a much later spectral type. However, the $\mathrm{NaI} D$ lines are very deep, indicating that this is due to a very large amount of interstellar reddening, which is confirmed by the extreme value of $E(B-V)$. The high probability of membership $(78 \%)$ of this star in the proper motion study by van Altena \& Jones (1972) makes it unlikely that this star is a background supergiant. Furthermore, luminosity class I characteristics should be recognizable in our spectrum in that case. When we look at the position of this star in the HR-diagram, we also note that it is located slightly above Palla \& Stahler's birthline for an accretion rate of $10^{-4} M_{\odot} \mathrm{yr}^{-1}$. This suggests that this star does indeed belong to the cluster, but might have already evolved off the main sequence. According to the evolutionary tracks by Maeder \& Meynet (1988), an object at this position in the HRD would be a $20 M_{\odot}$ star with an age of about 8 million years. In that case the extreme amount of reddening could be due to circumstellar material, possibly ejected by the central star.

74. At the position of this star, there seem to be two infrared sources. We identified the weaker one of the two in the $H$ band $(1.6 \mu \mathrm{m})$ and at longer wavelengths as the optical star, whereas the other could be an embedded source, not unlike the ones found in NGC 6611 (Hillenbrand et al. 1993). Furthermore, the optical component has $E(B-V)=0{ }^{\mathrm{m}} 15$, much smaller than the interstellar one to the cluster, suggesting it is a foreground object. When we look at this star's position in the HRD however, we conclude that its position agrees well with that of most NGC 6530 members. Also, it has a high (70\%) probability of cluster membership in the proper motion study by van Altena \& Jones (1972). Therefore, we conclude that maybe the optical colours of this star also contain a contribution of the embedded source, which results in an erroneous value of $E(B-V)$. More observations are necessary to clarify this issue.

79. This star is located to the right of the birthline in the cluster's HRD. Although the membership probability of this star in the proper motion study by van Altena \& Jones (1972) is relatively high $(69 \%)$, we conclude that most probably this star is a background giant. However, the possibility that it is a post-main sequence cluster member cannot be completely excluded.

114. $\mathrm{LkH} \alpha$ 108. In our IDS spectrum this star shows strong $\mathrm{H} \alpha$ emission, whereas all other lines are in absorption. Furthermore, it shows large $\left(>1^{\mathrm{m}}\right)$ photometric variations and a strong infrared excess. Volk \& Cohen (1989) present an IRAS LRS spectrum for $\mathrm{LkH} \alpha 108$ in which some weak silicate absorption features, as well as some PAH emission can be seen on a very red continuum. However, when we compare the flux level in this LRS spectrum with our SED, there seems to be too much flux in it to be due to $\mathrm{LkH} \alpha 108$ alone. The LRS spectrum is probably contaminated with other nearby infrared sources, making it difficult to draw any definite conclusions from it. If the PAH and silicate features are due to $\mathrm{LkH} \alpha 118$, this would point to it being a young stellar object, however. In the cluster's HRD $\mathrm{LkH} \alpha 108$ is located between the main sequence and the birthline for an accretion rate of $10^{-5} M_{\odot} \mathrm{yr}^{-1}$, close to the evolutionary track of a $5.5 M_{\odot}$ star. Since this star fulfills all of the criteria for membership of the Herbig Ae/Be stellar group as originally outlined by Herbig (1960) as well as all tested secondary criteria from Thé et al. (1994), we conclude that $\mathrm{LkH} \alpha 108$ must be a new Herbig Be star, which is probably physically located in NGC 6530 and younger than $10^{5}$ years.

118. The status of this object remains unclear. The star's position in the HRD, as well as the inconclusive membership probability (47\%), seem to suggest that this star is a foreground object. However, its $E(B-V)$ of 1.20 indicates that it is a background object. It could be that this star is an embedded foreground object or a multiple system.

W 9. HD 164816. This star is located slightly to the right of the main-sequence, in a region well above the birthline in the cluster's HRD. Since this O-type star is seen to ionize part of its surrounding hydrogen gas, this star is without any doubt a cluster member. Therefore, this star must already have evolved off the main-sequence. Further support for this view comes from the observation by Böhm-Vitense et al. (1984) that the $1549 \AA$ C IV line of $\mathrm{W} 9$ has a moderate $\mathrm{P}$ Cygni profile, indicative of mass loss. By comparing its position in the HRD with the postmain sequence evolutionary tracks by Maeder \& Meynet (1988), we conclude that HD 164816 is a $30 M_{\odot}$ star with an age of about $710^{6}$ years.

151. This star shows some moderate $(\approx 0.5)$ photometric variations, as well as probably some $\mathrm{H} \alpha$ emission and a moderate, but clearly visible, infrared excess. In the cluster's HR-diagram it is located close to the main sequence, near the evolutionary track of a $9 M_{\odot}$ star. Therefore we conclude that this star is a cluster member as well as a good new Herbig Be candidate, near the end of its pre-main sequence phase (age $>10^{5}$ years).

172. This star is located to the right of the birthline in the cluster's HRD, provided that the star is at the 
distance of NGC 6530. This, combined with the low (19\%) membership probability in the proper motion study by van Altena \& Jones (1972), indicates that this object is in fact not a cluster member. However, its $E(B-V)$ of 0.35 in Table 5 suggests that it is a cluster member. Since the equivalent width of its $\mathrm{Na}$ I $D$ lines indicate much larger amounts of gaseous material than those seen in most cluster members, we conclude that most probably this star is a background giant.

180. $\mathrm{LkH} \alpha$ 112. This star is a well-known Herbig Be star, with moderate $(\approx 0.5)$ photometric variations (Boesono et al. 1987; Shevchenko et al. 1993). Light curves and colour-magnitude diagrams of this star using data from literature are shown in Figs. 13a and 14a. In the light curve we can see pulse-like shapes very similar to the ones observed in the well-known Herbig Ae star HR 5999 (Pérez et al. 1992), which we understand now as being indicative of random instabilities in the line of sight due to a combination of phenomena such as extinction changes, bursts and clumpy accretion. From Fig. 14a we also note that there seems to be a weak correlation between $V$ and the $U-B, B-V$ and $V-R$ colours, suggesting that the observed variations are at least partly due to, presumably circumstellar, variable extinction. Our IDS spectrum shows very strong emission in $\mathrm{H} \alpha$ and $\mathrm{H} \beta$, whereas all other lines are in absorption. Furthermore, it displays a moderate infrared excess. In the cluster's HRD the star is located between the birthline for an accretion rate of $10^{-4} M_{\odot} \mathrm{yr}^{-1}$ and the main sequence, close to the evolutionary track of a $14 M_{\odot}$ star. We conclude that this cluster member is indeed a Herbig Be star, with an age of about $10^{5}$ years.

199. The $J H K$ magnitudes of this star seem to match its visual magnitudes poorly. The star does not appear to be variable, however. Therefore perhaps another star was measured in the near-IR observations.

201. HD 315031. When we look at the position of this star in the Hertzsprung-Russell diagram, we note that it is located above Palla \& Stahler's birthline for an accretion rate of $10^{-4} M_{\odot} \mathrm{yr}^{-1}$, which would suggest that this star is not a cluster member. The membership probability by van Altena \& Jones for this star (48\%) is not really conclusive, but the fact that this star has a similar polarization as other stars in NGC 6530 (McCall et al. 1990) suggest that it is in fact a cluster member. The deviating position of HD 310531 in the HRD is probably due to the fact that it is a binary with a secondary of the same spectral type as the primary (Boggs \& Böhm-Vitense 1989).

223. HD 315021. Böhm-Vitense et al. (1984) discovered both in- and outflowing material in the $1549 \AA \mathrm{C}$ IV and $1640 \AA$ He II line profiles of this star. In our HRdiagram it is located close to the birthline for an accretion rate of $10^{-4} M_{\odot} \mathrm{yr}^{-1}$, at the evolutionary track of a $15 M_{\odot}$ star. Therefore we conclude that most probably this star is a post-main sequence star, in which case its age would be about $1.510^{7}$ years according to the evolutionary tracks of Maeder \& Meynet (1988).

245. $\mathrm{LkH} \alpha$ 115. This star is a well-known Herbig Be star, with moderate $(\approx 0.4)$ photometric variations (Boesono et al. 1987; Shevchenko et al. 1993). Light curves and colour-magnitude diagrams of this star using data from literature are shown in Figs. 13b and 14b, respectively. From these figures we note the star definitely shows variations in both brightness and all colours, but there is no obvious correlation between magnitude and colour. Therefore, the observed variations cannot be due to variable circumstellar extinction. Since the colours also vary, the variations are probably also not due to an eclipsing companion. Perhaps the best possible explanation for the observed variability are variations in the accretion rate, not unlike those seen in many other Herbig and T Tauri stars (e.g. Herbst et al. 1994). Our IDS spectrum of LkH $\alpha$ 115 shows very strong emission in $\mathrm{H} \alpha$, as well as some weak emission in $\mathrm{H} \beta$, whereas all other lines are in absorption. Furthermore, the SED displays a moderate infrared excess. In the cluster's HRD it is located between the main sequence and the birthline for an accretion rate of $10^{-5} M_{\odot} \mathrm{yr}^{-1}$, close to the evolutionary track of a $5.5 M_{\odot}$ star. Therefore we conclude that $\mathrm{LkH} \alpha 115$ is indeed a Herbig Be cluster member, with an age of about $10^{5}$ years.

261. This star is located to the right of the birthline in the cluster's HRD. Although the membership probability of this star in the proper motion study by van Altena \& Jones (1972) is high $(75 \%)$, we conclude that most probably this star is a background giant.

270. HD 165052. According to its position in Fig. 11, this should be a very massive $\left(50 M_{\odot}\right)$ star, which has evolved slightly off the main-sequence. This view is again supported by the detection of outflowing material in the C IV line at $1549 \AA$ (Böhm-Vitense et al. 1984). According to the post-main sequence evolutionary tracks by Maeder \& Meynet (1988), its age should be about $310^{6}$ years in this case. However, since this is a binary system (Morrison \& Conti 1978), some deviations of the star's position in the HRD can be expected, making this age rather uncertain.

304. This star is located to the far right in the cluster's HRD, making it impossible for this star to be a pre-main sequence cluster member. At present it is not clear whether this star is a late-type background giant or a post-main sequence cluster member with circumstellar material, as seems to be the case for star No. 338. If the latter is the case, it would probably make it a post-AGB star with a mass of about $8 M_{\odot}$ and an age of about $410^{7}$ years.

315. The $J H K$ magnitudes of this star in the SED seem to match its visual magnitudes very poorly. The star does not appear to be variable, however. Therefore perhaps the wrong star was measured in the near-IR.

330. This star is located to the right of the birthline in the cluster's HRD. The membership probability by 



Fig. 13. a) Light curves of $\mathrm{LkH} \alpha 112$ in the Johnson photometric system. b) The same for $\mathrm{LkH} \alpha 115$
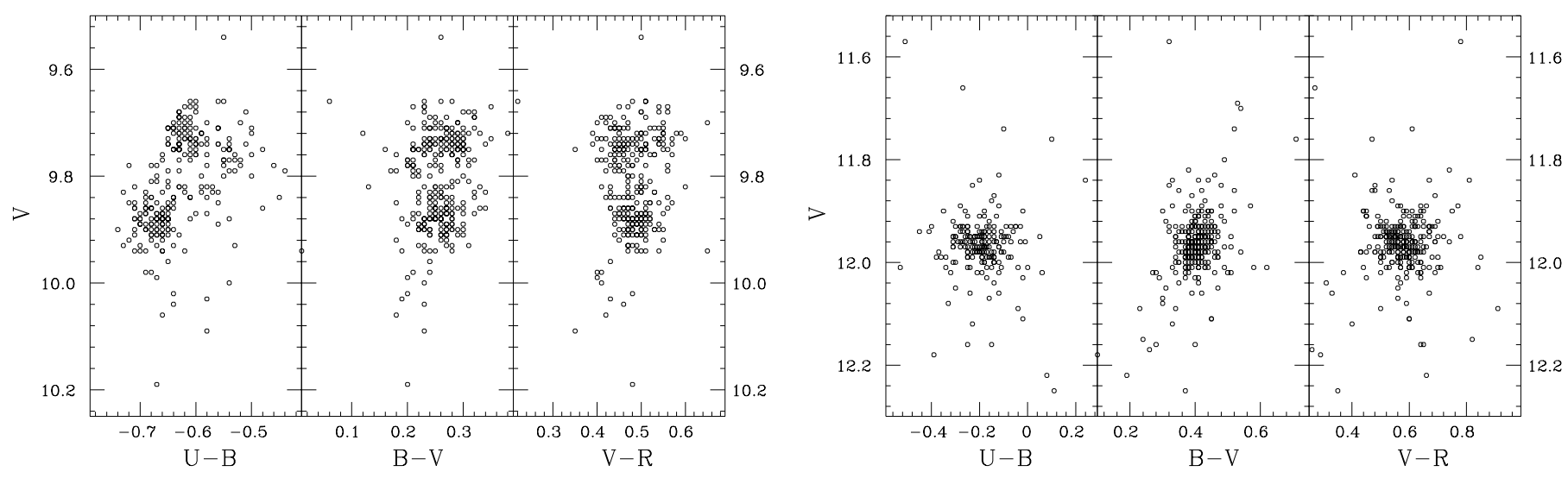

Fig. 14. a) Colour-magnitude diagrams of $V$ versus the Johnson $U-B, B-V$ and $V-R$ colour indices for LkH $\alpha 112$. b) The same for $\mathrm{LkH} \alpha 115$

van Altena \& Jones for this star $(48 \%)$ is not really conclusive. However, in our IDS spectrum of this star we can see B-type characteristics as well as traces of a cooler object. Therefore we conclude that this object is most probably a close binary, possibly located in NGC 6530 .

338. This star is located to the far right in the cluster's $\mathrm{HRD}$, making it impossible for this star to be a pre-main sequence cluster member. On first view this star seems to be a late-type background giant. However, in view of the high membership probability $(69 \%)$ of this star in the proper motion study by van Altena \& Jones (1972), the fact that this star has a similar polarization as other stars in NGC 6530 (McCall et al. 1990) and the fact that this star was marked as variable by Sagar \& Joshi (1978), the possibility of it being a post-main sequence cluster member with considerable amounts of circumstellar material should be seriously considered. Comparing this star's position in the HRD with the post-main sequence evolutionary tracks by Maeder \& Meynet (1988), we find that in such a case this star would be a $9 M_{\odot}$ star that has just evolved off the Asymptotic Giant Branch (AGB). Its age would be about about $310^{7}$ years. In view of the age of 
most stars in NGC 6530 this is fairly old, but still possible. If this scenario is correct, this would make it one of the youngest and most massive Post-AGB stars known. New observations, especially in the infrared, remain necessary to get more certainty on the true nature of this interesting object.

\section{Conclusions}

The distance and formation history of the very young open cluster NGC 6530 were investigated by studying a total of 132 probable member stars of this cluster. It was concluded that the distance towards NGC 6530 is $1.8 \pm 0.2 \mathrm{kpc}$, and its interstellar reddening $E(B-V)$ is 0.30 and more or less uniform across the cluster. Furthermore, it was concluded that the extinction law of the intracluster material is normal (i.e. $R_{V}=3.1$ ), but anomalous extinction laws were found for several more embedded stars in the cluster. No correlation between a star's position in the cluster with its extinction law could be found, demonstrating that these extinction laws are really due to circumstellar rather than intracluster material. This also shows that in the study of such very young open clusters the use of average extinction laws, as is done by some authors, is not correct; each star has its own individual extinction characteristics.

Of the 132 stars included in this study, 11 are suspected to be variable, five show $\mathrm{H} \alpha$ in emission and nine show an infrared excess. Three member stars of NGC 6530 $(\mathrm{LkH} \alpha 108, \mathrm{LkH} \alpha 112$ and $\mathrm{LkH} \alpha 115)$ were found to have strong infrared excesses as well as strong $\mathrm{H} \alpha$ emission. Furthermore, they are photometrically variable. From this we conclude that all three are most certainly members of the Herbig $\mathrm{Ae} / \mathrm{Be}$ stellar class. $\mathrm{LkH} \alpha 112$ and $\mathrm{LkH} \alpha$ 115 were already included in the most recent catalogue of Herbig Ae/Be stars (Thé et al. 1994), but $\mathrm{LkH} \alpha 108$ is a new Herbig Be star which deserves more attention. Two other programme stars (NGC 6530-45 and NGC 6530-151) also show strong infrared excesses and are photometrically variable. These two stars also show some moderate $\mathrm{H} \alpha$ emission, but the possibility that this is due to the surrounding nebulosity cannot be completely excluded. Therefore, we tentatively classify these stars as possible members of the Herbig Ae/Be stellar group. More research is necessary to give a more definite answer on the nature of these stars, however.

Herbig (1957) and Herbig \& Bell (1988) list several other emission line stars located in the field of NGC 6530 which were not included in our present study. The brighter of these have low membership probabilities in the proper motion study by van Altena \& Jones (1972) and are probably not physically associated with NGC 6530 . Following the model for the M8 region by Lada et al. (1976) and by Lightfoot et al. (1984) we propose that these are young stellar objects located deeper in the molecular cloud, and thus younger, than the stars in NGC 6530. No member- ship probability is known for the fainter $\mathrm{H} \alpha$ emitters in the field of NGC 6530 in the papers by Herbig (1957) and Herbig \& Bell (1988). Therefore, it is conceivable that in these stars we are seeing the first traces of a population of low mass pre-main sequence stars in NGC 6530. If this is really the case, this would give further support our conclusion that star formation in NGC 6530 has started longer ago than believed hitherto.

One probably cluster member (NGC 6530-338) was found to be located in a region of the HR-diagram located by post-AGB stars and is photometrically variable as well. Therefore we tentatively conclude that this star is a massive post-main sequence star that has just evolved off the Asymptotic Giant Branch. The same applies for another programme star (NGC 6530-304), but here no photometric variability was noted, making this very speculative. To confirm the post-AGB nature of both stars, new infrared data should be obtained to trace the dust shells these stars should have in this evolutionary stage.

We also constructed the luminosity function of NGC 6530, which was found to agree well with the theoretical models for cluster luminosity functions by Fletcher \& Stahler (1994). A bigger sample extending to less luminous stars is required to put these models through a more thorough test, however. Finally, from the distribution of preand post-main sequence stars in the cluster's HR-diagram, it was concluded that the process of star formation in NGC 6530 must have started a few times $10^{7}$ tears ago. For the low- and intermediate-mass stars this process is probably continuing up to the present day, whereas it must have already stopped for the massive stars. A further study of whether this process happened sequential or more or less simultaneous could have important implications for the further development of star formation theory.

Acknowledgements. The authors would like to thank E.A. Bibo for measuring some of the data in Table 2. This research has made use of the Simbad data base, operated at CDS, Strasbourg, France.

\section{References}

van Altena W.F., Jones B.F., 1972, A\&A 20, 425

Berrilli F., Corciulo G., Ingrosso G., Lorenzetti D., Nisini B., Strafella F., 1992, ApJ 398, 254

Bessell M.S., 1979, PASP 91, 589

Boesono B., Thé P.S., Tjin A Djie H.R.E., 1987, Ap\&SS 137, 167

Boggs D., Böhm-Vitense E., 1989, ApJ 339, 209

Böhm-Vitense E., Hodge P., Boggs D., 1984, ApJ 287, 825

Bouchet P., Monetti A., Slezak E., Le Bertre T., Manfroid J., 1989, A\&AS 80, 379

Brand J., Wouterloot J.G.A., 1988, A\&AS 75, 117

Buscombe W., 1984, Sixth General Catalogue of MK Spectral Classifications, Northwestern University, Evanston, Illinois

Chini R., Neckel Th., 1981, A\&A 102, 171

Conti P.S., Alschuler W.R., 1971, ApJ 170, 325

Fletcher A.B., Stahler S.W., 1994, ApJ 435, 329 
Fluks M.A., Plez B., Thé P.S., Westerlund B.E., 1996, A\&A (submitted)

Graham J.A., 1982, PASP 94, 244

Herbig G.H., 1957, ApJ 125, 634

Herbig G.H., 1960, ApJS 4, 337

Herbig G.H., Bell K.R., 1988, Lick Obs. Bull. 1111, 1

Herbst W., Herbst D.K., Grossman E.J., Weinstein D., 1994, AJ 108, 1906

Hester J.J., Scowen P.A., Sankrit R., et al., 1996, AJ 111, 2349

Hillenbrand L.A., Massey P., Strom S.E., Merrill K.M., 1993, AJ 106, 1906

Hiltner W.A., Morgan W.W., Neff J.S., 1965, ApJ 141, 183

Horne K., 1986, PASP 98, 609

Jacoby G.H., Hunter D.A., Christian C.A., 1984, ApJS 56, 257

Johnson H.L., 1966, ARA\&A 4, 193

Johnson H.L., 1967, ApJ 147, 192

Johnson H.L., Borgman J., 1963, B.A.N. 17, 115

Kilambi G.C., 1977, MNRAS 178, 423

Kurucz R.L., 1979, ApJS 40, 1

Kurucz R.L., 1991, Precision photometry: Astrophysics of the galaxy. In: Davis Philip A.G., Upgren A.R., Janes K.A. (eds.). L. Davis press, Schenectady, New York

Lada C.J., Gull T.R., Gottlieb C.A., Gottlieb E.W., 1976, AJ 203, 159

Lightfoot J.F., Deighton D.W., Furniss I., Glencross W.M., Hirst C.J., Jennings R.E., Poulter G., 1984, MNRAS 208, 197

Lub J., Pel J.W., 1977, A\&A 54, 137

Maeder A., Meynet G., 1988, A\&AS 76, 411
McCall M.L., Richer M.G., Visvanathan N., 1990, ApJ 357, 502

Morrison N.D., Conti P.S., 1978, ApJ 224, 558

Palla F., Stahler S.W., 1993, ApJ 418, 414

Pérez M.R., Thé P.S., Westerlund B.E., 1987, PASP 99, 1050

Pérez M.R., Webb J.R., Thé P.S., 1992, A\&A 257, 209

Pérez M.R., de Winter D., van den Ancker M.E., Grady C.A., Thé P.S., 1996, A\&A (in preparation)

de Ruiter H.R., Lub J., 1986, A\&AS 63, 59

Sagar R., Joshi U.C., 1978, MNRAS 184, 467

Schmidt-Kaler Th., 1982, Landölt-Bornstein Catalogue VI/2b

Shevchenko V.S., Grankin K.N., Ibragimov M.A., Melnikov S.Y., Yakubov S.D., 1993, Ap\&SS 202, 121

Stal'bovskii O.I., Shevchenko V.S., 1981, SvA 25, 25

Steenman H., Thé P.S., 1989, Ap\&SS 159, 189

Steenman H., Thé P.S., 1991, Ap\&SS 184, 9

Thé P.S., 1960, ApJ 132, 40

Thé P.S., Hageman T., Westerlund B.E., Tjin A Djie H.R.E., 1985, A\&A 151, 391

Thé P.S., de Winter D., Pérez M.R., 1994, A\&AS 104, 315

Torres A.V., 1987, ApJ 322, 949

Vázquez R.A., Feinstein A., 1992, A\&AS 92, 863

Volk K., Cohen M., 1989, AJ 98, 931

Walker M.F., 1957, ApJ 125, 636

Wesselius P.R., van Duinen R.J., de Jonge A.R.W., Aalders J.W.G., Luinge W., Wildeman K.J., 1982, A\&AS 49, 427

de Winter D., Koulis C., Thé P.S., van den Ancker M.E., Pérez M.R., 1996, A\&AS 121, 223 